\title{
EMC2: A versatile algorithm for robust tracking of calcium dynamics from individual neurons in behaving animals
}

\author{
Thibault Lagache ${ }^{1,2,3,4^{*}}$, Alison Hanson ${ }^{1,2,3,4}$, Jesús E. Pérez-Ortega ${ }^{1,2,3,4}$, \\ Adrienne Fairhall ${ }^{4,5,6,7}$ and Rafael Yuste ${ }^{1,2,3,4,8}$ \\ ${ }^{1}$ Department of Biological Sciences, Columbia University, New York, NY 10027 \\ ${ }^{2}$ Neurotechnology Center, Columbia University, New York, NY 10027 \\ ${ }^{3}$ Kavli Institute of Brain Science, Columbia University, New York, NY 10027 \\ ${ }^{4}$ Marine Biological Laboratory in Woods Hole, MA 02543 \\ ${ }^{5}$ Department of Physiology and Biophysics, University of Washington, Seattle, WA 98195 \\ ${ }^{6}$ UW Computational Neuroscience Center, University of Washington, Seattle, WA 98195 \\ ${ }^{7}$ WRF UW Institute for Neuroengineering, University of Washington, Seattle, WA 98195 \\ ${ }^{8}$ Donostia International Physics Center, DIPC, 20018 San Sebastian, Spain.
}

*Corresponding author: thibault.lagache@pasteur.fr. Present address: Institut Pasteur, Department of Cell Biology and Infection, 25 rue du Docteur Roux, 75015 Paris, France. 


\begin{abstract}
Measuring the activity of neuronal populations with calcium imaging can capture emergent functional properties of neuronal circuits with single cell resolution. However, the motion of freely behaving animals, together with the intermittent detectability of calcium sensors, can hinder automatic long-term monitoring of neuronal activity and the subsequent functional characterization of neural circuits. We report the development and open-source implementation of a multi-step cellular tracking algorithm (Elastic Motion Correction and Concatenation or $\mathrm{EMC}^{2}$ ) that compensates for the intermittent disappearance of moving neurons by integrating local deformation information from detectable neurons. We demonstrate the accuracy and versatility of our algorithm using calcium imaging data from two-photon volumetric microscopy in visual cortex of awake mice, and from confocal microscopy in behaving Hydra, which experiences major body deformation during its contractions. We quantify the performance of our algorithm using ground truth manual tracking of neurons, along with synthetic time-lapse sequences, covering a wide range of particle motions and detectability parameters. By monitoring the calcium activity of the same neuronal populations along several days in layer $2 / 3$ of visual cortex of mice, we report important turn-over within the active neurons across days, with only few neurons that remained active across days. Then, by combining automatic tracking of single neuron activity over long time-lapse sequences with statistical clustering, we characterize and map neuronal ensembles in behaving Hydra. We document the existence three major nonoverlapping ensembles of neurons (CB, RP1 and RP2) whose activity correlates with contractions and elongations. Our results prove that the $\mathrm{EMC}^{2}$ algorithm can be used as a robust platform for neuronal tracking in behaving animals.
\end{abstract}




\section{Introduction}

Tracking single neuron activity in freely behaving animals can help a detailed understanding of how neural circuits integrate external information, compute, learn and control animal behavior. Calcium imaging has become widespread for measuring single neuron activity as it is non-invasive and allows the simultaneous measurement of hundreds to thousands of cells, with single cell resolution [1]. Moreover, monitoring single neuron activity in freely moving animals such as rodents can be achieved with miniaturized microscopes attached to the head [2]. However, technical limitations of current microscopy techniques and of mathematical analysis hinder a more complete imaging and analysis of entire brains. Alternative strategies consist of monitoring single neuron activity in targeted brain regions of rodents using two-photon microscopy [3], or imaging the nervous system of a smaller animal, one that can fit entirely within a microscope's field of view, such as Caenorhabditis elegans [4], Hydra [5], Zebrafish [6] or Drosophila larvae [7]. Another advantage of simple model organisms is that they contain many fewer neurons than mammals and have a limited repertoire of behaviors [8] that could be entirely measured in a near future.

Aside from the difficulties in imaging of entire nervous systems with high temporal and spatial resolution [3, 6, 9], an important bottleneck in analyzing calcium imaging data is to achieve robust and automatic tracking of individual cells over long time-lapse sequences, while the animal is behaving. Single-cell tracking is challenging for three main reasons: First, there could be a large number of cells in a cluttered environment. Therefore, false positives and negatives during single cell detection and localization impede the association of detected neurons between successive time frames and call for more elaborate tracking algorithms. Second, neurons can remain undetectable over large periods of time because calcium sensors can be significantly brighter than background only if neurons are firing. Third, tracking methods in behaving animals have to handle animal motion and body deformations [10]. To tackle these issues, numerous hardware solutions have been proposed, including the fixation of animal (head) [3, 6, 11], the high-speed motorization of microscopes to track animal movements $[4,12]$ and the dual-color labeling of neurons with a calcium insensitive probe that can be detected and localized even when neurons are not firing [4, 9]. However, even when (some of) these hardware solutions are implemented, the residual motion of neurons, the limited spatial resolution of the microscope and the 
bioRxiv preprint doi: https://doi.org/10.1101/2020.06.22.165696; this version posted March 23, 2021. The copyright holder for this preprint (which was not certified by peer review) is the author/funder, who has granted bioRxiv a license to display the preprint in perpetuity. It is made available under aCC-BY-NC-ND 4.0 International license.

intermittency of fluorescence signal (when single-channel calcium imaging is used) hinder the robust tracking of single neuron activity. Therefore, elaborated postprocessing of acquired movies is required. Most of developed image processing methods consist of registering images (volumes) to reference image(s) (volume(s)) using either the local fluorescence intensity of images $[13,14]$, or directly the extracted neuron positions $[4,10,15,16]$. Then, additional image processing, such as nonnegative matrix factorization [17, 18], might be required for demixing and denoising cellular calcium activity.

Despite all these software developments, the difficult implementation of hardware solutions, such as the dual-labeling and imaging of calcium-insensitive probes, together with the important deformability of challenging animal models such as Hydra or Caenorhabditis elegans, prevent the robust automatic tracking of single neurons in many experiments. Tracking has then to be performed manually [5] or semimanually [19]. This limits the analysis to a few hundreds frames, introduces operator bias and, ultimately, hinders our understanding of the functional organization of nervous systems.

To robustly track particles with intermittent detectability (neurons) in a cluttered and deforming environment, we report the development of an algorithm named Elastic Motion Correction and Concatenation $\left(\mathrm{EMC}^{2}\right)$. In contrast to traditional single particle tracking methods, $\mathrm{EMC}^{2}$ does not set expected priors for particle motion (diffusion and/or linear motion typically). Instead, it uses information about local motion and deformation from detectable and tracked particles in the neighborhood of undetectable particles. $\mathrm{EMC}^{2}$ is therefore more versatile, and does not require motion priors or heuristics to close potentially long tracking gaps. For the local tracking of detectable particles, $E M C^{2}$ uses a probabilistic method and is therefore robust to very cluttered conditions. We validate the robustness and accuracy of $E \mathrm{MC}^{2}$ with manual tracking of neurons in calcium imaging of behaving animals. Our first dataset consists of twophoton calcium imaging of few tens of neurons in the visual cortex of awake mice. We show that our algorithm accurately tracks the limited motion of single neurons in the two-photon field-of-view, enabling the fast analysis of long recordings of individual neuron activity. We then monitor single neurons of Hydra's nervous system while the animal is behaving and deforming. Hydra imaging datasets represent perhaps the worst possible scenario for tracking purposes, since animals can have major changes in body size with non-isometric deformations. We also quantify the performance of 
bioRxiv preprint doi: https://doi.org/10.1101/2020.06.22.165696; this version posted March 23, 2021. The copyright holder for this preprint (which was not certified by peer review) is the author/funder, who has granted bioRxiv a license to display the preprint in perpetuity. It is made available under aCC-BY-NC-ND 4.0 International license.

Lagache et al., p.5

$E M C^{2}$ using simulations of fluorescence time-lapse sequences with different types of motion (confined diffusion, linear displacement and elastic deformation), and show that $\mathrm{EMC}^{2}$ outperforms state-of-the art tracking algorithms.

After integrating $\mathrm{EMC}^{2}$ in an open-source and freely available platform lcy [20] (icy.bioimageanalysis.org), we monitor the activity of single neurons in two-photon calcium imaging of layer $2 / 3$ of mice visual cortex over multiple days and show that there is an important turn-over in the active neurons from one day to another, and that actually, very few neurons remain active across days. We then track single neuron activity in behaving Hydra, and characterize the functional clustering of individual neurons into co-active ensembles [21]. Consistent with previous observations [5], we find that Hydra contains three main neuronal ensembles (CB, RP1 and RP2); and, after mapping the positions of individual neurons from each ensemble, we also confirm that these ensembles are not overlapping, i.e., they do not share neurons, and that they are correlated with contraction bursts and elongation behaviors.

These results demonstrate that $\mathrm{EMC}^{2}$ is an effective tracking algorithm for the long-term tracking of single neuron activity in calcium imaging of living animals. Robust tracking constitutes a prerequisite for the statistical analysis of the functional organization of neural circuits, the description of emergent computational units such as neuronal ensembles, and ultimately, for the understanding and prediction of animals' adaptive behavior.

\section{Results}

\section{1- Limitations of Single-Particle-Tracking Algorithms}

Most tracking algorithms rely on the automatic detection of particles (cells, molecules...) that are significantly brighter than the noisy background in each frame of the time-lapse sequence (see [22] and [23] for review) and, subsequently, the linking of detections between frames corresponding to the reconstruction of coherent particle trajectories (see Table 1). False positives (i.e. background signal) and negatives (i.e. missing detections) in the detection of particles, together with the influence of high particle density and stochastic dynamics has, over the last two decades, motivated the development of algorithms that go beyond naïve tracking methods that simply 
bioRxiv preprint doi: https://doi.org/10.1101/2020.06.22.165696; this version posted March 23, 2021. The copyright holder for this preprint (which was not certified by peer review) is the author/funder, who has granted bioRxiv a license to display the preprint in perpetuity. It is made available under aCC-BY-NC-ND 4.0 International license.

Lagache et al., p. 6

associate nearest-neighbor detections between consecutive time frames (see Table 1 and $[24,25]$ for a review of existing methods).

One category of elaborated tracking algorithms is based on global distance minimization (GDM) between all pairs of detections in consecutive time frames. The distance measure between detections can be simply the Euclidean distance, or can additionally take into account the similarity of the intensity and/or shape of the detected particle [26]. To handle possible missing and false detections, heuristics for track termination and initiation are defined by the user [27]. GDM methods are fast and robust, but user-defined parameters hinder their applicability in very cluttered conditions [24]. Moreover, the limitations of current particle motion models (confined diffusion and linear directed motion [26-28]) prevent the robust estimation of particle positions when they remain undetectable over long periods of time, as for sparsely firing neurons in calcium imaging. This limits the ability to close gaps in the trajectories of tracked particles to a very few frames.

Probabilistic methods are an attractive alternative to GDM methods, even if their computational cost is higher. Probabilistic algorithms model both the stochastic motion of the particles and their detectability, and then compute the optimal tracking solution by maximizing the model likelihood of observed detections [24, 29, 30]. The gold standard of probabilistic association is multiple hypothesis tracking (MHT) in which one computes all the possible tracking solutions over the entire time-lapse sequence, before inferring the tracking solution that maximizes the observation's likelihood. However, MHT is generally not computationally tractable. Approximate solutions that iteratively compute a nearly optimal solution over a limited number of frames (typically up to 5) have been proposed [24]. As probabilistic methods model the particles' detectability, they are usually more robust than GDM methods in cluttered conditions. However, the small number of frames considered when approximating the MHT solution together with limited particle motion models (again diffusion and/or linear displacements [24, 31]), reduce the capability of probabilistic algorithms to close large tracking gaps and keep track of particles' putative position when they remain undetectable over many frames.

\section{2- EMC ${ }^{2}$ Algorithm}


$E M C^{2}$ is a method and software to track single particles with intermittent detectability in cluttered environments. It is particularly well-suited for tracking single neuron activity with calcium imaging in a behaving animal. The $\mathrm{EMC}^{2}$ algorithm can be decomposed into four main steps (Figure 1).

First, bright spots (e.g. firing neurons in calcium imaging sequence) are automatically detected with a robust method based on the wavelet decomposition of the time-lapse sequence and the statistical thresholding of wavelet coefficients (Materials and Methods). Second, detected spots are linked into single particle trajectories with a state-of-the-art probabilistic algorithm, a variant of multiple hypothesis tracking (eMHT [24]), which is particularly robust in cluttered conditions. Obtained track(let)s correspond to trajectories of detectable particles. However, in many time-lapse sequences such as calcium imaging of neuron activity, tracks would be terminated prematurely when particles switch to an undetectable state (e.g. nonfiring neuron) and new tracks would be generated when particles can be detected again (e.g. firing neurons). This would create large time-gaps in individual tracks that need to be closed to allow the accurate tracking of each particle's identity over the time-lapse sequence. Thus, the two last steps of our method aim to close gaps in trajectories using information about the motion and deformation of the field of view along the time-lapse sequence. We considered that tracked particles are embedded in a deformable medium (e.g. neurons within tissue) and that local estimation of the deformation of the field-of-view should allow the inference of particles' positions even when they are undetectable.

The third step of $E M C^{2}$ is therefore the computation of the elastic deformation of the field-of-view at each time using the information contained in tracks of detectable particles. For this, we used the positions of tracked particles between consecutive time frames as fiducial source and target points. We then computed the forward and backward elastic deformations of the whole field by interpolating the deformation at any position between fiducials with a thin-plate-spline function. The thin-plate spline is a popular poly-harmonic spline whose robustness in image alignment and point-set matching has been demonstrated [32], and which has been recently applied for automatic neuron registration in time-lapse sequences [10]. We highlight that the Neuron Registration Vector Encoding (NeRVE) method developed to map single neurons in C. elegans [10] is not sufficiently robust for tracking neurons in calcium imaging of behaving Hydra as showed in [15]. The poor tracking accuracy of NerVE 
method when applied to Hydra is due to two main reasons. The first reason is that, contrary to $C$. elegans worm, one cannot map the Hydra neuron positions in cylindrical coordinates along the principal axis of the animal. This drastically reduces the effective deformation of $C$. elegans and thus, the complexity of neuron registration. The second main reason is the intermittent detectability of neurons in calcium imaging, compared to the calcium-insensitive RFP labeling used in [10]. Indeed, calcium imaging is related to neuron activity and leads to tracking gaps with longer and more variable lengths. Altogether, the higher animal deformation and the stochastic detectability of neurons result in an important error-propagation during neuron registration. This can explain the low accuracy of the global vector clustering method of the NeRVE algorithm when applied to Hydra [15]. To increase the robustness and accuracy of single neuron tracking, we rather implement, in $\mathrm{EMC}^{2}$, a local concatenation of short tracks (tracklets) after having propagated forward and backward the estimated deformation of the field of view.

Therefore, the fourth and last step of our method is the iterative estimation and correction of the elastic deformation of the field-of-view, followed by the optimal concatenation of tracklets. In this last step, we used the elastic transformation computed with thin-plate splines to propagate forward (and backward) the putative positions of undetectable particles, following the termination of their detectable tracklets (or preceding the initiation of novel tracklets). After having corrected for the elastic deformation of the field-of-view, we then linked short tracklets by minimizing the global distance between the end-points of prematurely terminated tracklets with the starting-points of newly appearing tracklets (Materials and Methods). Finally, singleparticle tracks over the time-lapse sequence are obtained by applying the computed elastic transformation to the concatenated tracklets. 


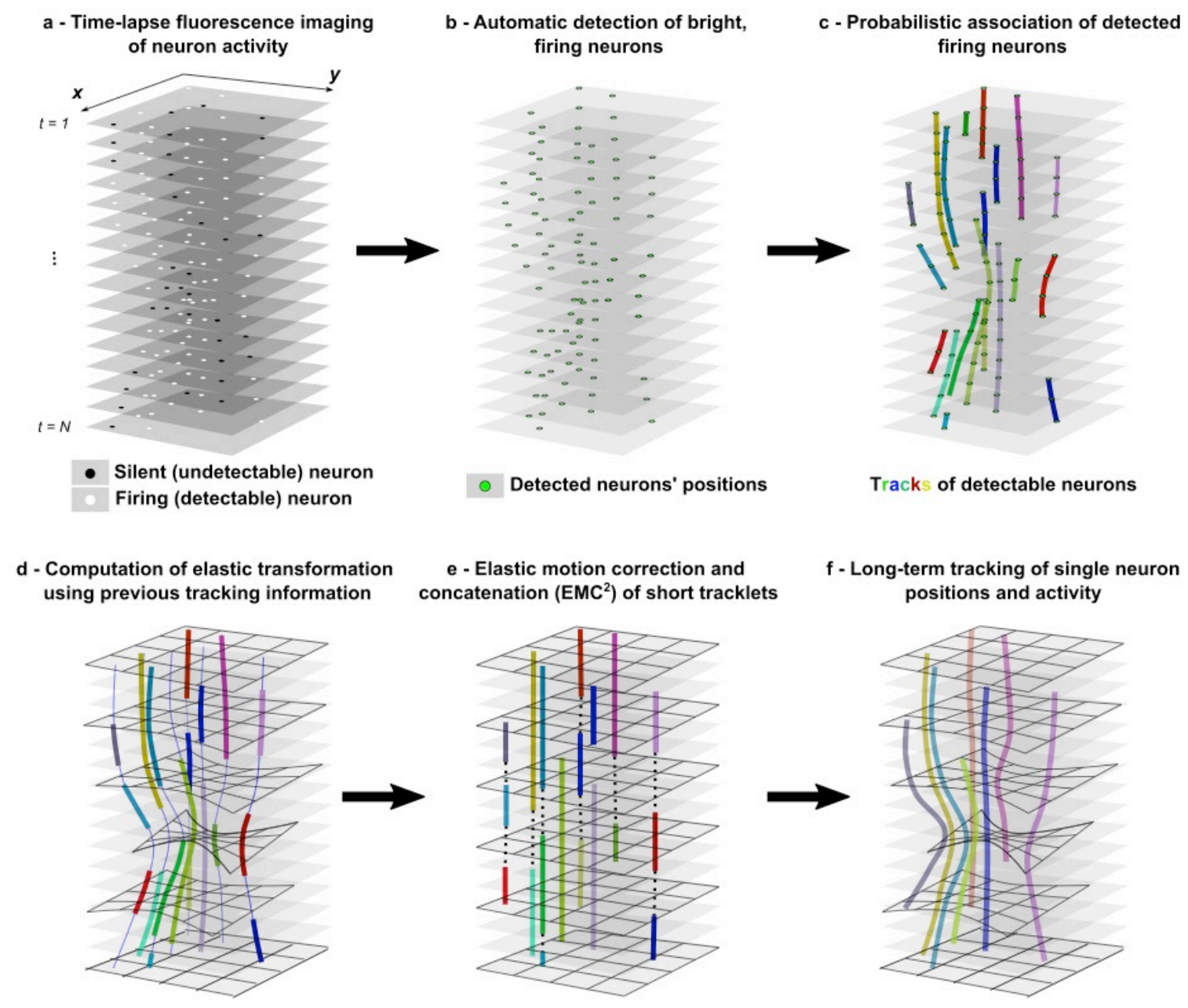

Figure 1: Multi-step EMC ${ }^{2}$ for tracking single neuron activity in calcium imaging data. aTime-lapse imaging ( $N$ frames) of intermittent fluorescence activity of a neuron in a deforming environment (e.g. behaving animal). b- Fluorescent spots (neurons), that are significantly brighter than background, are automatically detected with a wavelet-based algorithm. cTracklets of detectable neurons are robustly reconstructed using probabilistic tracking algorithm (eMHT). $\boldsymbol{d}$-Short tracklets of detectable particles are used to compute the elastic deformation of the field of view at each time frame. Associated detections in neuron tracklets are used as fiducials, and the whole deformation is interpolated using a poly-harmonic thinplate spline function. Forward-and backward-propagated positions of tracklet particle positions are shown with a thin blue line. e- After having corrected for the deformation of the field-ofview where neurons are embedded, gaps between the end- and starting-points of tracklets are closed by minimizing the global Euclidean distance between points (dotted line). f- Finally, complete single neuron tracks over the time-lapse sequence are obtained by applying the elastic transformation of the field-of-view to concatenated tracklets. 
Contrary to gap-closing GDM approaches [27], EMC ${ }^{2}$ contains only two free parameter: the maximal distance between forward propagated end-points and backward-propagated starting-points of short tracklets for concatenation, and, to avoid important error propagation, a maximum time-lag between ending- and starting-point candidates. We highlight that concatenated tracklets do not necessarily span the entire time lapse sequence: each track begins with the first detection of its first concatenated tracklet and ends with its last detection of the last concatenated tracklet. Moreover, $E^{2} C^{2}$ algorithm handles complex natural motions and deformations, contrary to GDM methods that only account for confined or linear motion. $\mathrm{EMC}^{2}$ is therefore more robust and versatile. For the sake of reproducibility and dissemination of our method, we implemented the $\mathrm{EMC}^{2}$ multi-step procedure in the bio-image analysis software suite Icy [20] (http://icy.bioimageanalysis.org/). Icy is an open-source platform that is particularly well-suited for multi-step analysis thanks to graphical programming (plugin protocols) where each step of the analysis can be implemented as a block with inputs and outputs that can be linked to the other blocks (Figure 2 and Materials and Methods). Our method builds on well-established Icy preprocessing functions for spot detection and tracking. 


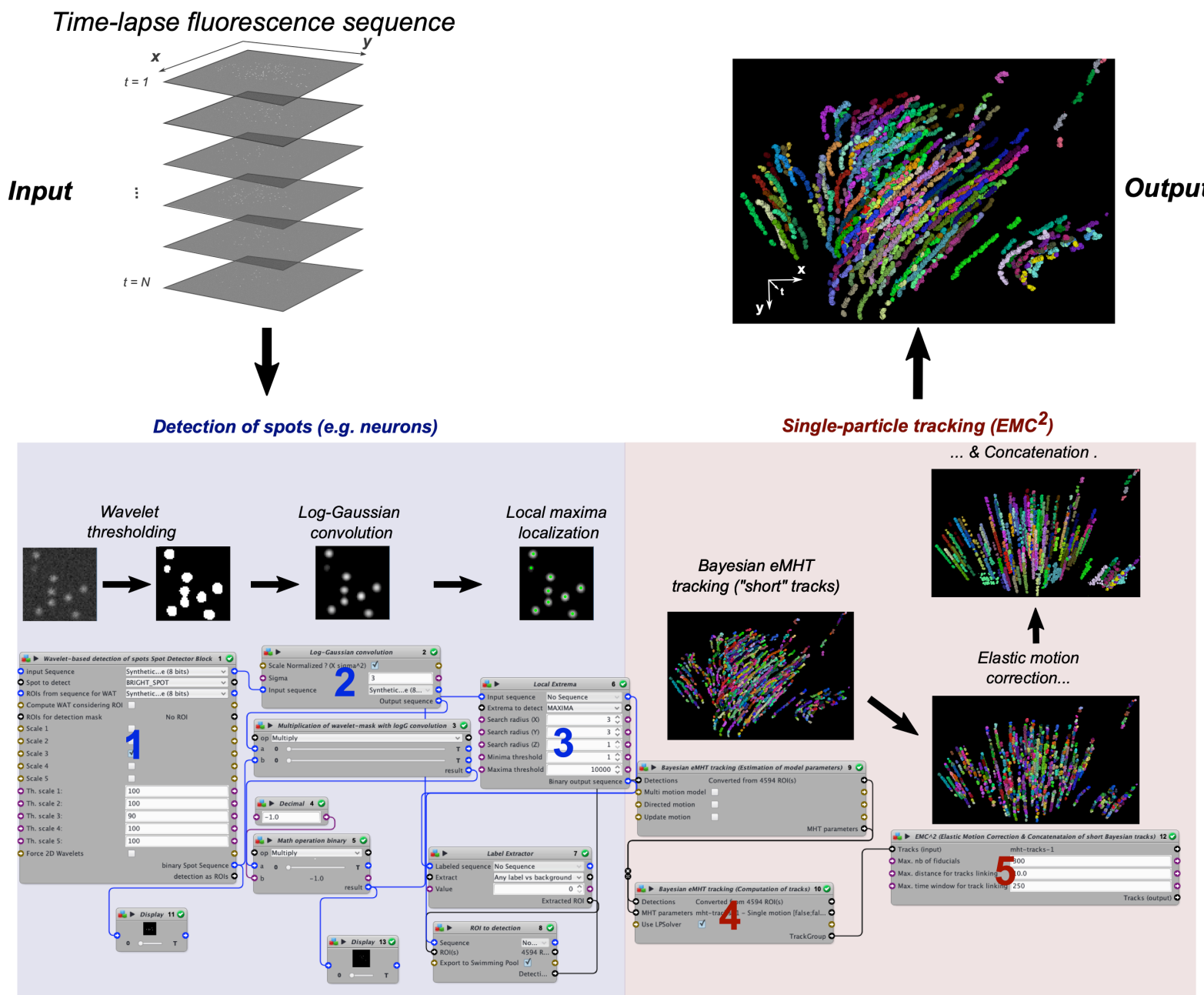

Figure 2: Implementation of the EMC ${ }^{2}$ algorithm in Icy platform. Time-lapse sequence of fluorescent particles is input to a multi-step, automatic protocol in lcy. A first series of blocks, highlighted in blue, detects the position of fluorescent neurons (spots) in each frame of the time-lapse sequence. Block 1 uses the wavelet transform of each image and statistical thresholding of wavelet coefficients to determine spots that are significantly brighter than background. To separate close spots that form clusters in the wavelet-based mask of the image, the thresholded sequence is convolved with a log-gaussian kernel to enhance single spots (block 2), and local maxima algorithm is applied (block 3). A second series of blocks, highlighted in red, computes single particle tracks from computed spot positions. First, the Bayesian tracking algorithm (eMHT) computes tracklets of detectable particles (block 4). Due to fluctuating particle detectability, many Bayesian tracklets are terminated prematurely and new tracklets are created when particles can be detected again. To close detection gaps in single particle tracks, block 5 applies the EMC ${ }^{2}$ algorithm. Final output of the lcy protocol is the collection of single particle tracks over the time-lapse sequence. Tracking protocol can be found here: http://icy.bioimageanalysis.org/protocol/detection-with-cluster-un-mixing-andtracking-of-neurons-with-emc2/ and is also directly accessible through the search bar of the Icy software (see step-by-step Supplementary Icy tutorial). 


\section{3- Validation of $E M C^{2}$}

a- Manual tracking of calcium dataset in behaving animals

To validate the capabilities of $\mathrm{EMC}^{2}$, we first compared the results of our algorithm with manual tracking in calcium imaging sequences of neuron activity in behaving Hydra [5]. We used the first 250 frames of a time-lapse sequence previously acquired in a genetically-engineered animal [5] and automatically detected the active neurons (bright spots) using the multi-step detection process described in the Materials and Methods. We tracked the detected particles with the eMHT algorithm ([24], implemented in Spot tracking plugin in Icy) and obtained short Bayesian tracklets ( $\mathrm{n}=$ 784 tracks) for the detected neurons (step 4 of the Icy protocol in Fig. 2). We then manually concatenated all the corresponding tracklets, i.e. we closed gaps, and obtained complete neuron tracks ( $n=444$ tracks). We observed that, before gap closing, tracklets were significantly shorter than concatenated tracks, meaning that many tracklets are indeed terminated prematurely by the undetectability of silent (nonfiring) neurons. We measured the accuracy of $\mathrm{EMC}^{2}$ by comparing the computed tracks with those obtained after manual association of tracklets, which we took as an approximate ground truth (see Materials and Methods and Supplementary Figure 2). We also measured how tracks obtained with TrackMate [27] implemented in Fiji [28] matched this manual ground truth. TrackMate is a GDM method, based on optimal linear assignment between closest detections. To handle the gaps in tracking when particles are undetectable, TrackMate again uses a GDM algorithm to compute the optimal linear assignment between end- and starting-points of previously computed tracks. As a result, TrackMate uses the same type of gap closing algorithm as EMC 2 but without correcting for potential elastic deformation of the field-of-view. Finally, to evaluate how well the algorithm performs to generate the initial set of short tracklets, i.e. to compare the probabilistic eMHT used in $\mathrm{EMC}^{2}$ with the GDM algorithm used in TrackMate, we also measured the accuracy of $\mathrm{EMC}^{2}$, but without elastic motion correction before gap closing. Compared algorithms are summarized in Table 2. First, we found that $\mathrm{EMC}^{2}$ ( $\mathrm{n}=453$ tracks, with 410 (90.5\%) matched tracks) outperformed TrackMate $\left(n=474\right.$ tracks, with $259(54.6 \%)$ matched tracks) and $\mathrm{EMC}^{2}$ without elastic 
correction ( $n=514$ tracks, with $279(54.3 \%)$ matched tracks). The similar capabilities of TrackMate and $\mathrm{EMC}^{2}$ without elastic motion correction indicate that Bayesian eMHT and the GDM tracking method perform similarly for local association of detectable spots, but fail at closing longer tracking gaps in deformable media. This highlights the importance of elastic motion correction before the optimal concatenation of short tracks.

Using the same methodology, we compared manual and automatic $\left(E M C^{2}\right)$ tracking of single neurons in the more trivial case of two-photon imaging of the visual cortex (layer 2/3) of mice (we used the first day calcium recording from the first animal [33]). Here, the deformation of the field-of-view is much more limited and the motion of embedded neurons can be assimilated to confined diffusion. As expected, we obtained an $\mathrm{EMC}^{2}$ accuracy that was close to $100 \%$ (64 correct tracks over 65 , i.e. $98.5 \%$ accuracy) for a time lapse sequence of 3,700 frames (5 minutes).

b- Synthetic time-lapse sequences

Manual gap closing in time-lapse sequences is tedious and prone to operator bias. Moreover, the ground truth, i.e. the identity of each individual neuron along the whole time-lapse sequence, is unknown. Therefore, we designed a reproducible, synthetic approach where we simulated individual neurons' activity and animal motion with different sets of parameters.

We modeled three different types of motion and/or deformation (Materials \& Methods): Confined diffusion, where blinking neurons diffuse within a confined area (as in two-photon imaging of targeted brain areas), Linear motion where neurons all move together in the same direction at constant velocity, and finally, using deformation fields measured in Hydra experimental data, we simulated naturalistic Hydra deformations. We further modeled the intermittent activity of neuronal ensembles with a probabilistic Poisson model. We also modeled the fluorescence dynamics of individual spikes using a parametric curve that we fitted to experimental data. Finally, using neuron positions, firing activity and fluorescence dynamics, we generated synthetic time-lapse sequences using a mixed Poisson-Gaussian noise model ([24] and Materials \& Methods).

For confined diffusion (150 simulated tracks, $\mathrm{n}=10$ simulations), both $\mathrm{EMC}^{2}$ and TrackMate gave excellent results, with track matches of $93.5 \% \pm 1.6 \%(144.4 \pm 1.8$ 
correct tracks over $154.6 \pm 0.9$ reconstructed tracks) for $\mathrm{EMC}^{2}$ and $92.9 \% \pm 0.8 \%$ for TrackMate (140.3 \pm 1.0 correct tracks over $151.0 \pm 0.3$ reconstructed tracks) (Fig.3a). The good performance of TrackMate was expected as this algorithm was initially designed to track confined endocytic spots at the cell membrane [27]. In addition to confined motion, TrackMate can also model linear motion of particles when computing the optimal gap closing between short tracks. Therefore, in linear motion simulations (337.6 \pm 1.0 simulated tracks, $n=10$ simulations), we used TrackMate with linear motion correction instead of standard confined motion correction. However, even with linear correction, the performance of Trackmate $(76.3 \% \pm 0.6 \%(262.6 \pm 1.4$ correct tracks over 358.2.0 \pm 1.8 reconstructed tracks)) was significantly worse than EMC2's performance $(97.7 \pm 0.5 \%$ (326.1 \pm 1.6 correct tracks over $337.3 \pm 1.1$ reconstructed tracks)). This difference is due to the different estimation methods that are used in the two tracking algorithms to estimate the direction of tracks: in TrackMate, the estimation of track directions is local, based on the last detection within each short track, whereas the estimation of track direction in $\mathrm{EMC}^{2}$ uses global information provided by neighbouring short tracks and is therefore more robust. Finally, in the third case, we used the deformation field that we estimated over 250 frames within a time-lapse experimental sequence of Hydra (500 simulated tracks, $n=10$ simulations, see section 2.a and Materials \& Methods). We found that Trackmate had similar performances with (matching score $69.4 \pm 1.1 \%$ (414.8 \pm 3.7 correct tracks over $598.2 \pm 4.8$ reconstructed tracks)) or without $(72.4 \pm 0.9 \%(427.2 \pm 3.0$ correct tracks over 590.7 \pm 3.5 reconstructed tracks)) linear motion correction, and that both were outperformed by $\mathrm{EMC}^{2}(98.6 \pm 0.3 \%(493.4 \pm 1.6$ correct tracks over $500.4 \pm 0.2$ reconstructed tracks)).

We also measured the robustness of $\mathrm{EMC}^{2}$ to parameter change in synthetic simulations. In particular, we measured the performance of the algorithm for an increased percentage of stable cells (Fig. 3.d) (see Material and Methods), an increased number of neurons (Fig. 3.e) and an increased length of simulated sequences (Fig. 3.f). First, we found that even without stable cells ( $\left.\alpha_{\text {stable }}=0\right)$, the accuracy of EMC ${ }^{2}$ remained high $(77.7 \% \pm 3.6 \%$ (398.8 \pm 18.4 correct tracks over $513.3 \pm 1.7$ reconstructed tracks), and rapidly increased to $91.8 \% \pm 1.2 \%$ for $\alpha_{\text {stable }}=$ $5 \%$, before reaching a plateau above $95 \%$ accuracy for $\alpha_{\text {stable }} \geq 10 \%$. Conversely, we found that $\mathrm{EMC}^{2}$ was very sensitive to the number of neurons, with poor performance 
for very few neurons (for simulations with only 10 neurons, the accuracy was $11.6 \%$ $\pm 0.6 \%$ (5.6 \pm 0.3 correct tracks over $49.8 \pm 0.8$ reconstructed tracks $))$. The accuracy rapidly increased to $>90 \%$ when more than 100 neurons were simulated. Tracking errors in simulations with few neurons are due to the inaccurate estimation of the deformation field, and iterative error propagation, when few fiducial source and target points are used. Finally, we measured how the performance of the EMC ${ }^{2}$ decreased with the length of the simulated sequence, for three different animals (animal 1, 2 and 6 see Table 3). We chose animals with highest number of neurons so that we could accurately estimate, and therefore simulate, their body deformation. We found that the $E^{2}$ accuracy slowly decreased with the sequence length but remained high even for longer sequences. Indeed, for simulated sequences, with 500 simulated neurons, from the estimated deformation of animal 1 (resp. 2 and 6), the accuracy decreased from $98.6 \% \pm 0.3 \%$ (resp. $91.8 \% \pm 0.3 \%$ and $97.8 \% \pm 0.3 \%$ ) for simulated sequences with 250 frames, to $87.5 \% \pm 0.8 \%$ (resp. $85.5 \% \pm 0.4 \%$ and $96.9 \% \pm$ $0.3 \%$ ) for 500 frames and $81.8 \% \pm 1.1 \%$ (resp. $73.4 \% \pm 1.1 \%$ and $92.0 \% \pm 1.3 \%$ ) for either 900 (animal 1) or 1000 (animal 2 and 6) frames. We also highlight that, in addition to high accuracy, the number of reconstructed tracks remained close to 500 even for longer sequence, with $500.4 \pm 0.16$ (250 frames), $511.7 \pm 0.99$ (500 frames) and $515.2 \pm 0.98$ (900 frames) reconstructed tracks for animal 1, $511.5 \pm 1.25$ (250 frames), $518.9 \pm 1.06$ (500 frames) and $542.7 \pm 3.25$ (1000 frames) reconstructed tracks for animal 2, and $502.9 \pm 0.67$ (250 frames), $505.6 \pm 0.72$ (500 frames) and $515.7 \pm 2.3$ (1000 frames) reconstructed tracks for animal 6 .

The sustained performance of $\mathrm{EMC}^{2}$ for long time-lapse sequences is a particularly desirable characteristic of a tracking algorithm as it allows the robust analysis of single neuron activity and functional coupling during different animal behaviors. Altogether these simulations show that $\mathrm{EMC}^{2}$ is a robust tracking algorithm, independent of the type and complexity of particle motion, and is therefore a versatile method for single particle tracking. 
a-

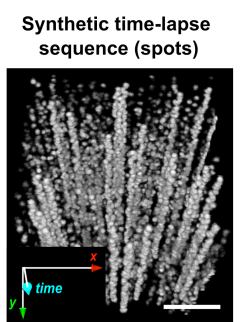

Simulated Tracks

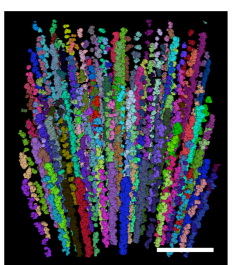

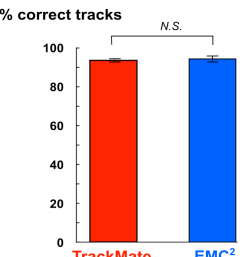

b-
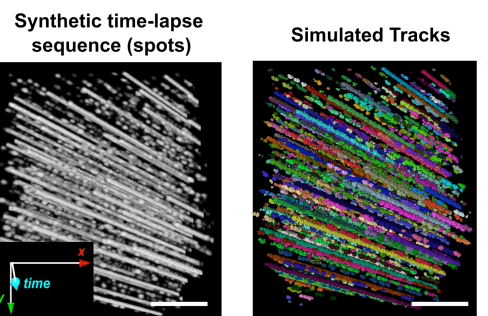

Tracking results

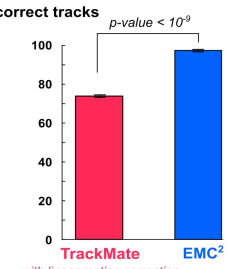

c-
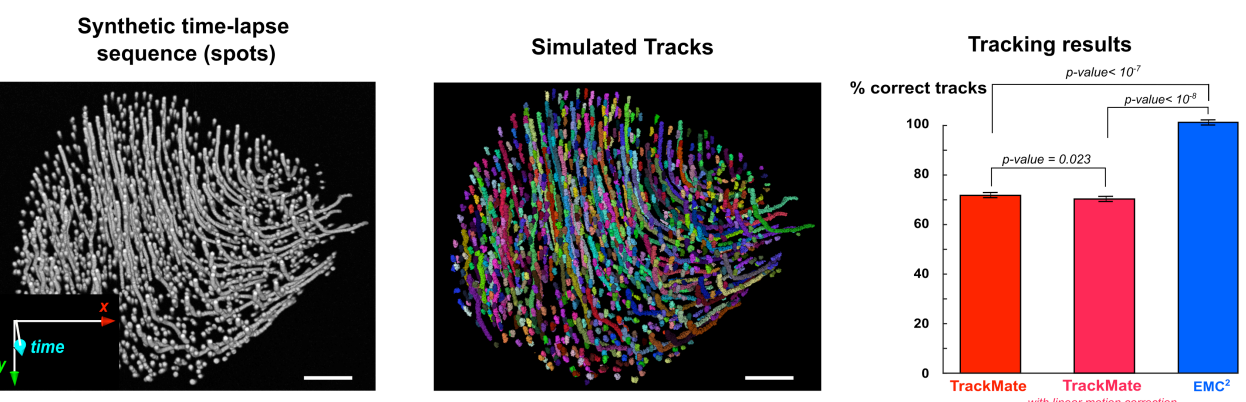

d-

e-

f-
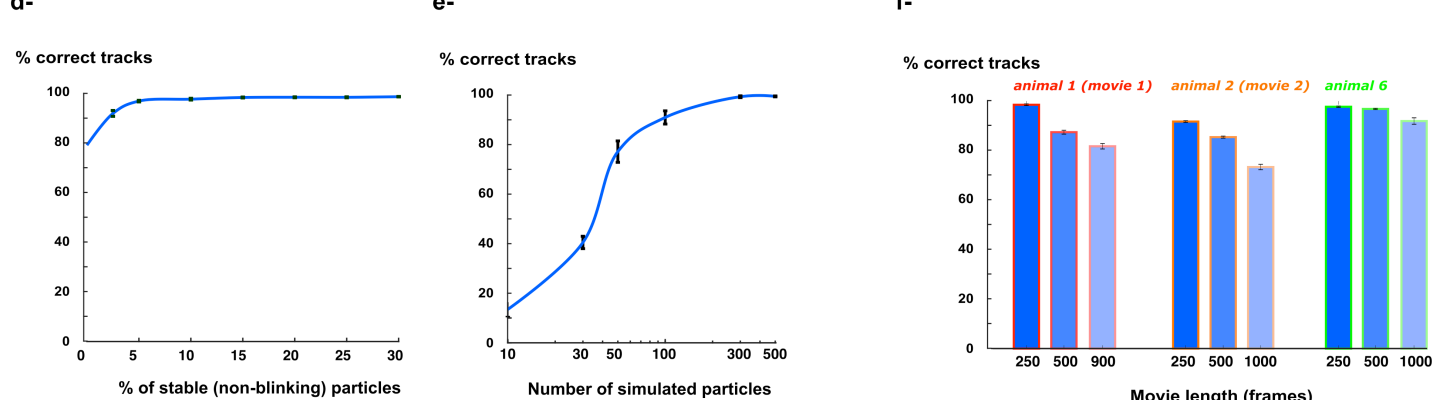

Figure 3: Testing EMC ${ }^{2}$ robustness with synthetic simulations. For each simulated type of motion (confined diffusion (a), linear motion (b) and "Hydra-like" elastic deformation (c)), we simulated the stochastic firing of neuronal ensembles and corresponding fluorescence dynamics in synthetic time-lapse sequences (see Materials and Methods for details). We then compared the performances of EMC ${ }^{2}$ (blue) with TrackMate (no motion correction (red) or linear motion correction (magenta)). P-values are obtained with the Wilcoxon rank sum test over $n=10$ simulations in each case. (d-e) Using "Hydra-like" synthetic deformation, we measured the accuracy of EMC ${ }^{2}$ for increasing proportion of stable (i.e. non-blinking) particles (neuronal cells) and increasing number of simulated particles. (f) After having estimated the deformation-field in three different animals (animal 1 (red), 2 (orange) and 6 (green)), we measured the accuracy of $E M C^{2}$ for simulated sequences with increasing length (250, 500 and 900 or 1000 frames).

4- Tracking and analyzing single neuron activity in behaving animals

a. Monitoring neuron activity with two-photon microscopy in mouse cortex 
Two-photon calcium imaging is widely used to monitor single neuron activity in targeted brain regions of awake and behaving animals [3]. The head of the animal is usually fixed under the objective of the microscope, which limits the motion of neurons and facilitates their individual tracking. However, residual neuron motion due to animal movements, breathing or heart beating requires computational post-processing of acquired time-lapse sequences to robustly monitor calcium activity of single neurons. The most popular technique for (slight) motion correction in time-lapse sequences is the elastic registration of fluorescence images with respect to one (or multiple) reference frame(s) using the image intensity [13]. The computational load of image registration is important, especially for long sequences with large images, and algorithms have been developed to speed-up the registration process and decrease the computational time to few minutes for $\sim 2,000$ frames time-lapse sequences (with 256x256 images) [14]. After image registration, the segmentation of neuronal masks for calcium fluorescence monitoring is then performed with standard intensity thresholding [33] or more elaborated techniques when neuronal masks are overlapping, such as non-negative matrix factorization [17].

To simultaneously localize and correct for neuron motion, we applied the EMC ${ }^{2}$ Icy tracking protocol (Fig. 2) to two-photon time-lapse calcium images of mouse visual cortex (Fig. 4 and Material and Methods). Imaging was performed during 5 minutes at $12.3 \mathrm{~Hz}\left(\sim 3,700\right.$ frame sequences) at days 1, 2 and 46 . The entire EMC ${ }^{2}$ protocol with neuron spot detection and tracking for each $\sim 3,700$ frame sequence ran in $\sim 3$ minutes with a 2,7 GHz Intel Core i7 processor. Consistently with [33], we observed an important turn-over of active neurons (Fig. 4a) with few neurons (median $=22 \%$ (15/68 neurons), $\mathrm{n}=4$ animals) that remained active all the days. We also observed a similar number of active neurons at day 1 and 2, but a decreased number at day 46 which is probably due to several factors such as decreased transgene expression or repeated experimental procedures [33]. We then analyzed single neuron trajectories obtained with $\mathrm{EMC}^{2}$ (Fig. 4b). Even if the head of the animals were fixed under the microscope, residual motion of the field-of-view led to confined stochastic trajectories for single neurons. The positions of single neurons at each time were either computed with the intensity center of detected spots when neurons were detectable, or estimated using the computed elastic deformation of the field of view when neurons were silent and undetectable. We measured a median neuron displacement between frames of $\sim 0.25$ pixels, and a median maximum distance of excursion (relatively to the center point of 
the trajectory) of $\sim 4$ pixels (Fig. $4 b$ ).

Altogether, these results show that $\mathrm{EMC}^{2}$ tracking protocol is a robust and fast method to post-process two-photon calcium imaging from awake animals. Trajectory analysis revealed the stochastic confined motion of single neuron positions, even in head-fixed animals. This residual motion is partly due to the animal movements, but also to the uncertainty of sub-pixel localization of neuron spots at each time frame [34]. Moreover, the analysis of single neuron activity across days in the same cortical region showed an important turn-over within the pool of active neurons each day, with few neurons remaining active all days. Statistical analysis recently showed that this latter pool of active neurons could be a stable neuronal ensemble [33].

\section{a- Two-photon calcium imaging \& tracking with EMC ${ }^{2}$}
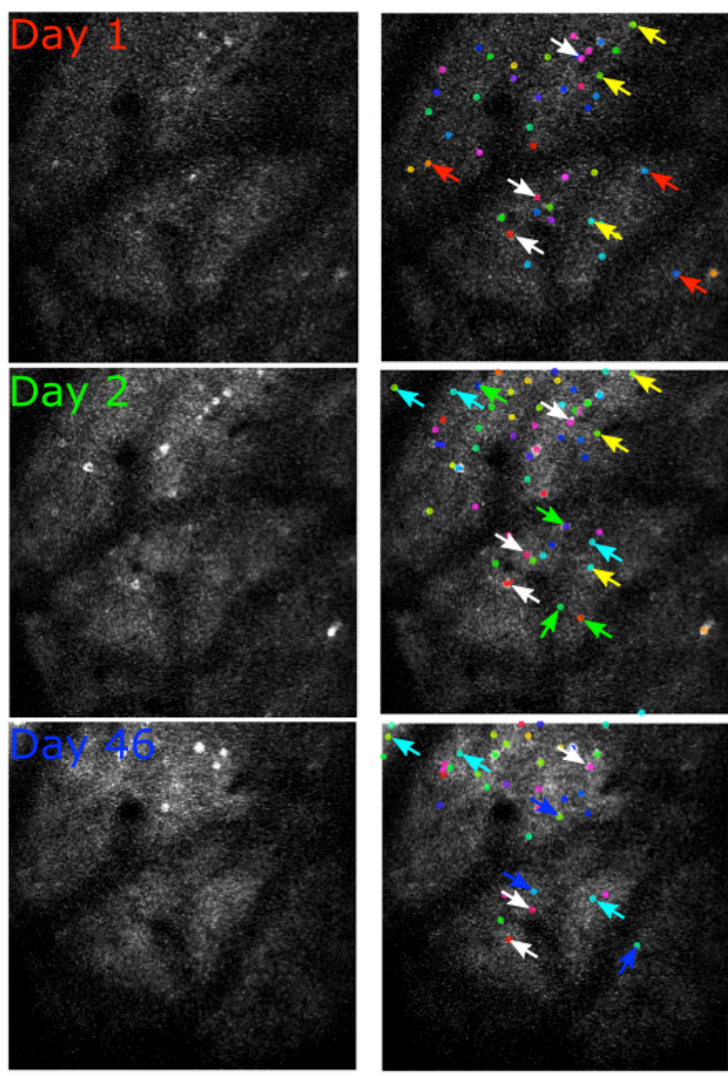
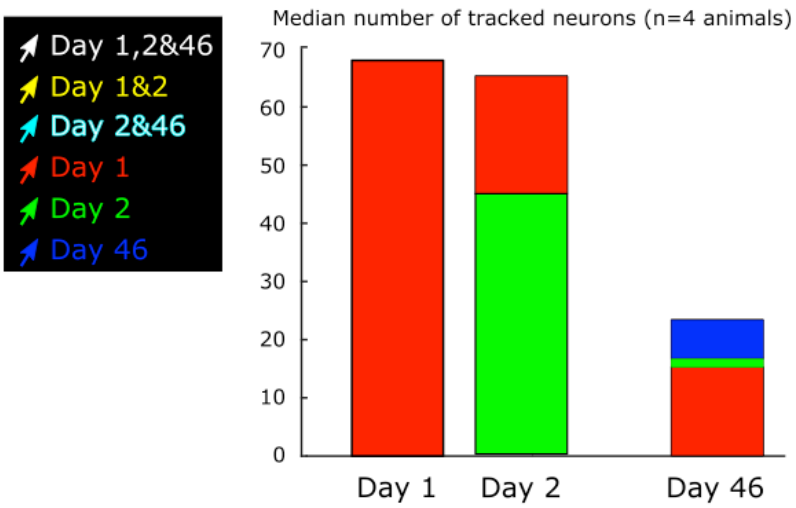

b- Confined neuron trajectories
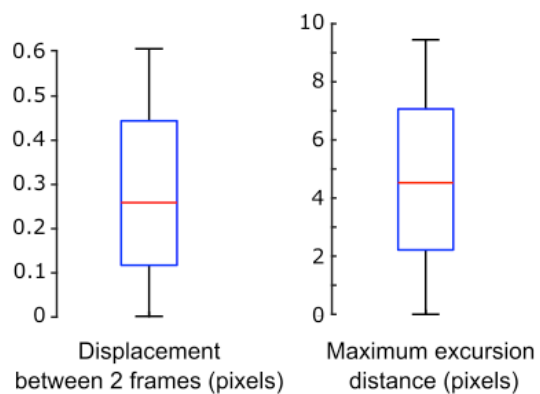

Figure 4: Monitoring the activity of individual neurons in two-photon calcium imaging of mouse visual cortex with EMC ${ }^{2}$. a- Two-photon calcium imaging of single neuron activity in visual cortex of awake mice is performed at Day 1, Day 2 and Day 46 during 5 minutes at 12.3 Hz. Tracking of neuron positions is performed with EMC ${ }^{2}$ and reveals an important turnover of active neurons across days. Examples of neurons that are active at Day 1, Day 2 or Day 46 are respectively highlighted with red, green or blue arrows. Neurons active at Day $1 \& 2$ are highlighted with yellow arrows, at Day $2 \& 46$ with cyan arrows, and at Day 1,2\&46 with 
bioRxiv preprint doi: https://doi.org/10.1101/2020.06.22.165696; this version posted March 23, 2021. The copyright holder for this preprint (which was not certified by peer review) is the author/funder, who has granted bioRxiv a license to display the preprint in perpetuity. It is made available under aCC-BY-NC-ND 4.0 International license.

white arrows. The median number of active neurons each day is also plotted ( $n=4$ animals). The number of neurons that are active from Day 1, 2 or 3 are respectively represented in red, green or blue. $\boldsymbol{b}$ - Single neuron trajectories can be modeled with confined stochastic motion. Two example trajectories are shown (green \& blue trajectories) with a maximum excursion distance of $\sim 4$ pixels. Boxplots of single neuron displacement between two consecutive frames, and maximum excursion distance (in pixels) are plotted ( $n=590$ trajectories).

\section{b. Characterizing neuronal ensembles in behaving Hydra}

There is increasing experimental evidence that neurons are organized into neuronal ensembles composed of a few tens of highly coupled neurons, and that these co-active ensembles are the fundamental computational units of the brain rather than single neurons themselves [21, 35]. Using manual annotation, it has been shown that Hydra's nervous system, one of the simplest of the animal kingdom, may be dominated by three main functional networks that extend through the entire animal $[5,36]$. To confirm (or refute) these observations, we used $\mathrm{EMC}^{2}$ and automatically tracked single neurons in $n=13$ time-lapse sequences (length $1067 \pm 56$ frames at $10 \mathrm{~Hz}$ (Materials and Methods) from 8 different animals (Table 3 and Fig. 5). Movies used in this study (avi and tiff files) can be downloaded from the BioStudies platform (https://www.ebi.ac.uk/biostudies/studies/S-BSST428). Analyzed movies were significantly longer than the manually annotated one (length $=200$ frames at $10 \mathrm{~Hz}$ [5]). Ensemble activity, corresponding to the co-firing of neurons, can be detected as significant peaks within the raster plot of single neuron activity (Fig. 5 and Materials and Methods). We detected a mean number of $17 \pm 2.2$ peaks per movie, corresponding to a mean rate of 1 activity peak every 63 frames (6.3 s) which corresponds well with the 4 peaks observed previously in the 200 frame movie [5]. To associate each peak with a putative neuronal ensemble, we adopted a similar approach as in [37] and measured the similarity between these events in terms of the identities of the participating neurons (Fig. 5 and Materials and Methods). We then performed k-means clustering of peak similarity, and used the Silhouette criterion [38] to determine the most likely number of neuronal ensembles causing the detected peaks of activity. We found 2 or 3 neuronal ensembles in each movie ( 3 neuronal ensembles were detected in 3 out of the 13 movies (23\%)). We then categorized each detected ensemble into one of the previously defined ensembles $[5,36]$ : Contraction 
Burst (CB) neurons that fire during longitudinal contraction of the animal, Rhythmic Potential 1 (RP1) that fire during the longitudinal elongation of the animal, and Rhythmic Potential 2 (RP2) neurons that fire independently of RP1 and CB activity. We found CB neuronal ensembles in almost all movies (11/13 (85\%) movies) and all animals (8/8 (100\%)), RP1 ensembles in all movies and animals and RP2 ensembles in fewer movies (5/13 (38\%)) and only 2/8 (25\%) animals. The absence of detected CB ensembles in 2 movies corresponds to the observed absence of contraction cycles within these movies. On the other hand, we hypothesize that the absence of RP2 ensembles in 6/8 (75\%) of the animals is due to the limited depth of the field-of-view in confocal microscopy (see Materials and Methods). Indeed, RP2 neurons lie in the thin ectoderm of the animal [5] that may not have been imaged in some animals. Finally, we classified and mapped each individual neuron in the detected ensembles (see Materials and Methods). As in [5], we found that the CB ensemble was the most important group of neurons with a mean number of $82 \pm 26$ neurons representing $18.7 \% \pm 4.4 \%$ of the total number of neurons (a mean of $374 \pm 53$ neurons were tracked over $>150$ frames in the different movies). The RP1 ensemble, with $43 \pm 12$ neurons $(10.8 \% \pm 2.3 \%)$, was the next largest ensemble and RP2, with $38 \pm 8$ (7.6 \pm $1.8 \%)$, was the third. The relative number of neurons in the different ensembles is in agreement with previous observations [5]. However, the overall size of each ensemble is smaller than the size reported previously. This is due to the fact that automatic classification of each individual neuron in an ensemble is more stringent than the manual classification that had been previously performed. Finally, we measured the overlap between ensembles, i.e. the proportion of single neurons that belonged to more than one ensemble. For each pair of ensembles, we computed the ratio between the number of shared neurons and the total number of neurons in both ensembles. We obtained ratio equal to $2.1 \% \pm 0.7 \%, 2.4 \% \pm 0.4 \%$ and $1.6 \% \pm 0.6 \%$ respectively for CB-RP1, CB-RP2 and RP1-RP2 ensemble overlap. These very low values confirm the non-overlap of main neuronal ensembles in Hydra [5]. By coupling the automatic tracking of individual neurons in multiple Hydra with robust clustering analysis of neuronal activity, we were therefore able to confirm the previous observations that the Hydra nervous system is dominated by three main non-overlapping ensembles that are involved in different animal behaviors. 


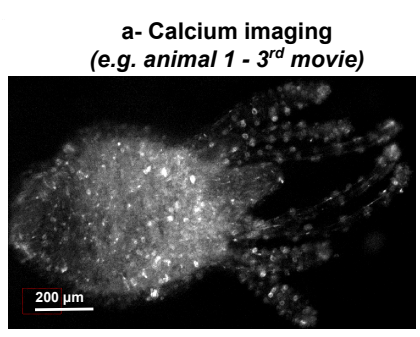

f- Neuronal ensembles

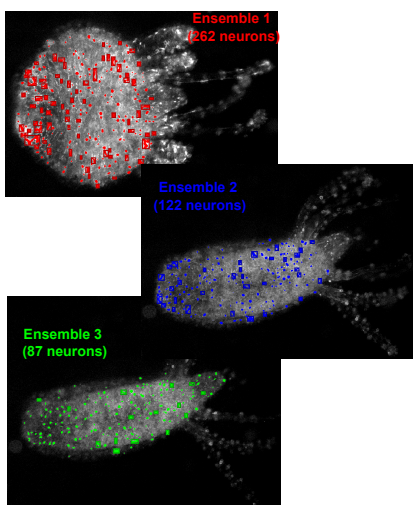

b- Single neuron tracks
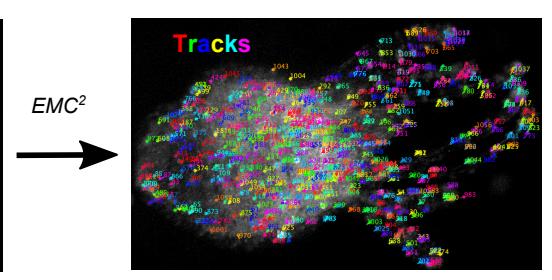

e- Peaks clusters

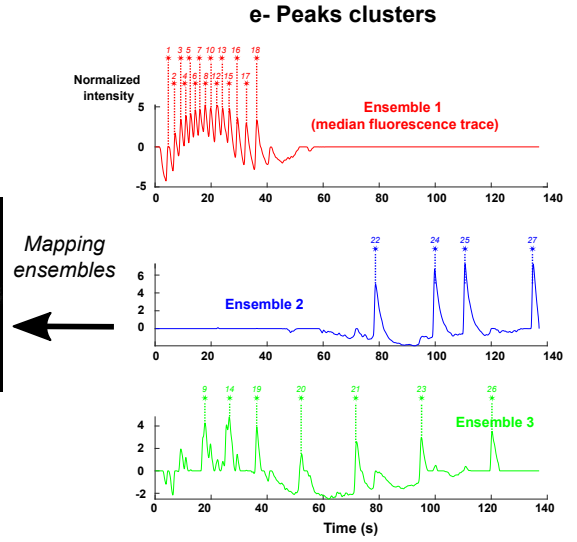

c- Raster plot of single neuron activity

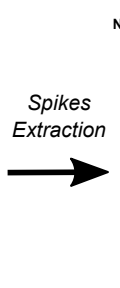
Activity peaks (ensembles)

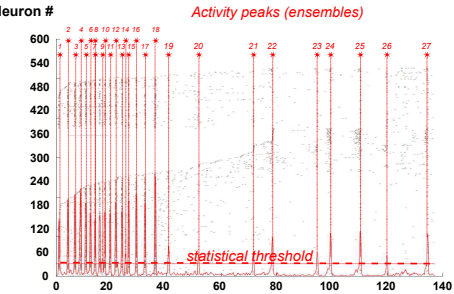

\rfloor$_{\text {Similarity }}^{\text {analysis }}$

d- Similarity between activity peaks (individual neurons involved)

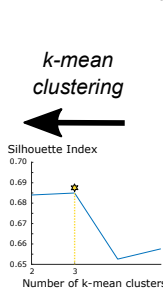
20

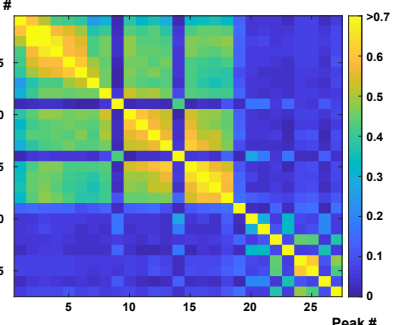

Figure 5: Single neuron tracking and mapping of neuronal ensembles in behaving Hydra. a- Calcium imaging of single neuron activity in behaving Hydra. The images and analysis of the $3^{\text {rd }}$ movie of animal 1 are given as representative examples. $\boldsymbol{b}$ - Single neuron tracks and fluorescence intensity are obtained with $E M C^{2}$ algorithm. c- For each neuron, spikes are extracted from fluorescence traces. Peaks of activity (highlighted with red stars) correspond to significant co-activity of individual neurons (sum of individual activities (solid red line) > statistical threshold (dashed red line), $p=0.001$ see Materials and Methods). Each peak putatively corresponds to the activation of one neuronal ensemble. $\boldsymbol{d}$ - Similarity between activity peaks is computed using the identities of individual neurons that are firing at each peak (see Materials and Methods). e- The optimal number of peak classes (that putatively correspond to the number of neuronal ensembles) is computed using the Silhouette index on k-means clustering of the similarity matrix (see Material and Methods). Median fluorescence trace of each neuronal ensemble and corresponding activity peaks are shown. The classification of individual neurons in each ensemble is determined based on their firing at ensemble peaks (see Materials and Methods). $\mathrm{f}$-Individual neurons of each ensemble can be dynamically mapped in the original time-lapse sequence.

Altogether, these results show that $\mathrm{EMC}^{2}$ is sufficiently robust to monitor single neuron activity over long periods of time in behaving animals. This constitutes a fundamental prerequisite for the analysis of neurons' functional organization and, ultimately, for our understanding of their emergent computational properties. 


\section{Discussion}

When using calcium imaging in living animals, an important challenge is the sustained tracking of neuronal positions over long periods of time in a moving, and potentially also deformable, environment. To tackle this issue, we have developed an algorithm, $\mathrm{EMC}^{2}$, that tracks detectable particles with a state-of-the-art probabilistic tracking algorithm that uses the information contained in reconstructed tracks about the local deformation of the field-of-view (e.g. animal) to estimate the position of undetectable particles and potentially close long tracking gaps. We validated the performance and versatility of $\mathrm{EMC}^{2}$ by comparing its performance with a state-of-theart tracking algorithm on manually tracked neurons in time-lapse calcium-imaging of behaving animals, including imaging over days of the same neurons from mouse visual cortex, the challenging deformable Hydra, and also on synthetic time lapse sequences that modeled different types of motion/deformation of the field-of-view (confined diffusion, linear motion and Hydra-like elastic deformation). In all cases, EMC ${ }^{2}$ showed high accuracy and outperformed state-of-the-art tracking methods.

Compared to traditional tracking approaches, composed only of particle detection and linking (see Table I), our hybrid algorithm is better equipped to handle long tracking gaps and general particle motion. Recently, tracking methods based on artificial neural networks $[39,40]$ have been introduced to handle more general particle motion, not only diffusion and/or linear motion. However, these methods are designed to link two sets of particle detections in consecutive time frames and, even if they can handle some missing or false detections, they are not well suited for long tracking gaps such as those encountered in calcium imaging of neuronal activity.

On-going development of high-speed three-dimensional microscopes enable the imaging of neuronal activity with high temporal and spatial resolution in an increasing number of animal models $[6,9]$. In a near future, the extension of EMC ${ }^{2}$ tracking method to three-dimension movies should not present important technical issues. Indeed, the thin-plate-spline transform used here to estimate the deformation of the field-of-view in two-dimensions is actually a special case of polyharmonic splines that have been specifically designed for the robust interpolation between data points in any dimensional space[41]. 
After having implemented $\mathrm{EMC}^{2}$ in the open-source bio-imaging platform Icy [20], we tracked single neuron activity in behaving mouse visual cortex and also Hydra over long time-lapse sequences ( 1000 frames at $10 \mathrm{~Hz}$, see table 3). In mouse cortex, our algorithm, performed essentially flawlessly, and reveal the presence of neurons that continue to respond to the same stimulus over several days. In addition, using statistical clustering, we confirmed the previous observations that neural activity of Hydra is dominated by three major, non-overlapping neuronal ensembles that are involved in the animal's repetitive contraction and elongation. At the same time, automatic clustering of neuronal activity was not able to extract other smaller ensembles of the animal's nervous system, such as the tentacle and sub-tentacle ensembles [5] that we could observe by eye. Indeed, the coordinated activity of small ensembles is difficult to detect from individual neuronal spiking because of sparse activity and noise. Moreover, the activation of these smaller ensembles is less frequent than the activation of the three major ensembles and happened in only few movies. Therefore, the complete mapping of neuronal ensembles of Hydra, with the characterization of even the smallest neuronal ensembles with less frequent activity, will require in the near future further development of better imaging and tracking of single neuron activity over longer periods of time.

To conclude, our results show that $\mathrm{EMC}^{2}$ is a robust and versatile tracking algorithm that allows monitoring and quantification of single neuron activity in behaving animals over long periods of time. Robust tracking of neural activity is a first step towards a better understanding of the neural code, i.e. how connected neuronal ensembles integrate information, trigger adaptive behavior and, more generally, compute the animal's behavioral or internal states. 


\section{MATERIALS AND METHODS}

\section{1- Elastic motion correction and concatenation $\left(E M C^{2}\right)$ of short tracks}

The eMHT algorithm returns a set of $N$ short tracks (tracklets) $\tau_{i}$, for $1 \leq i \leq N$, of detectable particles; the $i^{\text {th }}$ tracklet $\tau_{i}$ starts at position $x_{i}\left(t_{i}^{S}\right)$ at time $t_{i}^{S}$ and ends at position $\boldsymbol{x}_{i}\left(t_{i}^{e}\right)$ at time $t_{i}^{e}$. To concatenate tracklets, i.e. to link the tracklets that putatively correspond to the same neuron, we then estimate the backward position $\widehat{\boldsymbol{x}}_{\boldsymbol{i}}^{\boldsymbol{b}}(t)$ of the particle (neuron) corresponding to the $i^{\text {th }}$ tracklet before the starting-point $\left(t \leq t_{i}^{S}\right)$ of the tracklet. To estimate $\widehat{\boldsymbol{x}}_{\boldsymbol{i}}^{\boldsymbol{b}}(t)(t)$ for $t \leq t_{i}^{S}$, we iteratively apply backward thin-plate-spline transformation $\widehat{\boldsymbol{x}}_{\boldsymbol{i}}^{\boldsymbol{b}}(t-1)=T P S_{\text {backward }}\left(\widehat{\boldsymbol{x}}_{\boldsymbol{i}}^{\boldsymbol{b}}(t)\right)$, with the initial condition $\widehat{\boldsymbol{x}}_{\boldsymbol{i}}^{\boldsymbol{b}}\left(t_{i}^{S}\right)=\boldsymbol{x}_{i}\left(t_{i}^{S}\right)$. Similarly, to estimate the forward position $\widehat{\boldsymbol{x}}_{\boldsymbol{i}}^{f}(t)$ after the ending-point of the tracklet, i.e. for $t \geq t_{i}^{e}$, we iteratively apply the forward transformation $\widehat{\boldsymbol{x}}_{\boldsymbol{i}}^{f}(t)=T P S_{\text {forward }}\left(\widehat{\boldsymbol{x}}_{\boldsymbol{i}}^{f}(t-1)\right)$ with the initial condition $\widehat{\boldsymbol{x}}_{\boldsymbol{i}}^{f}\left(t_{i}^{e}\right)=\boldsymbol{x}_{\boldsymbol{i}}\left(t_{i}^{e}\right)$. From the estimated positions of particles corresponding to each tracklet, we then compute the distance $d_{i, j}$ between tracklets $\tau_{i} \neq \tau_{j}$ with

$$
d_{i, j}=\left\{\begin{array}{c}
\infty, \text { if } t_{i}^{e}>t_{j}^{s} \text { or } t_{j}^{e}>t_{i}^{s} \\
\min _{\mathrm{t}_{\mathrm{i}} \leq \mathrm{t} \leq \mathrm{t}_{\mathrm{j}}^{\mathrm{s}}}\left\|\left[\widehat{\boldsymbol{x}}_{i}^{f}-\widehat{\boldsymbol{x}}_{j}^{b}\right](t)\right\|, \text { if } t_{i}^{e} \leq t_{j}^{S} \leq t_{i}^{e}+g a p_{\max } \\
\min _{\mathrm{t}_{\mathrm{j}}^{\mathrm{e}} \leq \mathrm{t} \leq \mathrm{t}_{\mathrm{i}}^{\mathrm{s}}}\left\|\left[\widehat{\boldsymbol{x}}_{j}^{f}-\widehat{\boldsymbol{x}}_{i}^{b}\right](t)\right\|, \text { if } t_{j}^{e} \leq t_{i}^{S} \leq t_{j}^{e}+\operatorname{gap}_{\max }
\end{array}\right.
$$

where $\operatorname{gap}_{\max }$ is a user-defined maximum time gap that $\mathrm{EMC}^{2}$ is allowed to close (typically a few hundreds of frames). We need to apply a maximum time gap for timelapse sequences with particles that remain undetectable over long period of time due to the error growth during backward/forward estimation of the putative position of undetectable particles.

Using the computed distances between tracklets, we then define an association cost matrix,

$$
\boldsymbol{\Phi}=\left(\begin{array}{ccc}
\phi_{1,1} & \cdots & \phi_{1, N} \\
\vdots & \ddots & \vdots \\
\phi_{N, 1} & \cdots & \phi_{N, N}
\end{array}\right)
$$

with $\phi_{i, j}=d_{i, j}$ if $d_{i, j}<d_{\max }$, and $\phi_{i, j}=\infty$ otherwise. $d_{\max }$ is the second user-defined parameter of our tracking algorithm that specifies the maximum distance allowed between the forward-propagated end-point of a tracklet and the backward-propagated 
starting-point of another tracklet. Finally, among all possible associations for which the cost $\phi_{i, j}<\infty$, the optimum set of concatenated tracklets $\tau_{i^{*}} \rightarrow \tau_{j^{*}}$ among all the tracklets $\left\{\tau_{i}, \tau_{j}\right\}, 1 \leq i, j \leq N$ is the solution of the global minimization problem

$$
i^{*} \rightarrow j^{*}=\min _{i, j \text { such that } \phi_{i, j}<\infty} \sum \phi_{i, j}
$$

This minimization problem, known as the assignment problem, is similar to the problem solved in GDM methods of tracking, where algorithms determine the optimal association between particle detections by minimizing the global distance between detections in consecutive time frames of the sequence. One of the first and most popular algorithms to solve assignment problems is the Hungarian algorithm [42]. However, due to its computational load, faster algorithms have been proposed over the years. We used here the Jonker-Volgenant algorithm [43] implemented in the TrackMate plugin in ImageJ (see table 1).

Finally each particle (neuron) track $T_{i}$ for $1 \leq i \leq N^{\prime}$, with $N^{\prime} \leq N$ the number of "long" tracks, results from the concatenation of $n_{i}$ tracklets: $T_{i}=\tau_{i_{1}} \rightarrow \tau_{i_{2}} \rightarrow \cdots \rightarrow \tau_{i_{n_{i}}}$ with $t_{i_{1}}^{e} \leq t_{i_{2}}^{S} \leq \cdots \leq t_{i_{n_{i}}}^{S}$. We highlight that, by construction, each long track $T_{i}$ does not necessarily span over the whole time-lapse imaging sequence, but begins at the starting-time $t_{i_{1}}^{S}$ of the first tracklet $\tau_{i}$ and ends at the ending-time $t_{i_{n_{i}}}^{e}$ of the last tracklet $\tau_{i_{n_{i}}}$

\section{2- Icy protocol}

\section{a- Detection of spots (e.g. neurons) in time-lapse sequences}

To detect automatically the positions of fluorescent spots, corresponding to detectable particles, in each frame of the time lapse sequence, we designed a multistep algorithm (see Fig. 2) where we first detected fluorescent spots that are significantly brighter than background with a fast and robust algorithm based on a wavelet transformation of the image and statistical thresholding of the wavelet coefficients (block number 1 in Icy protocol (Fig. 2)) [44]. These spots correspond to individual particles or clusters of particles (e.g. neurons). To separate individual particles in the detected clusters, we then multiplied the original sequence with the binary mask obtained with wavelet thresholding and convolved the result of the multiplication with a log-Gaussian transformation (block number 2). The log-Gaussian 
convolution is similar to the point-spread function of microscopes and thus enhances individual particles [45]. Finally, we extracted the positions of single particles by applying a local-maxima algorithm (block number 3 ) to the convolved sequence.

\section{b- Single-particle tracking (EMC $\left.{ }^{2}\right)$}

After having detected the positions of fluorescent spots in each time frame, a second series of blocks computed the tracks of each single particle. First, block number 4 used the positions of spots and a robust Bayesian algorithm (eMHT [24]) to compute single tracks of detectable particles. Due to fluctuating detectability, many computed tracks are terminated prematurely and new tracks are created when particles are detectable again. We thus applied EMC ${ }^{2}$ algorithm (block number 5) to close gaps and reconstruct single-particle tracks over the entire time lapse sequence.

Tracking protocol can be found here: http://icy.bioimageanalysis.org/protocol/detection-with-cluster-un-mixing-and-

tracking-of-neurons-with-emc2/ and is also directly accessible through the search bar of the Icy software. Step-by-step tutorial for tracking neurons and exporting track intensity with Icy is provided as Supplementary material.

\section{3- Validation of EMC ${ }^{2}$}

\section{a- Comparison metric}

To compare the tracks obtained with $\mathrm{EMC}^{2}$ and other automatic tracking algorithms with ground truth tracks, we first considered the whole set of detections $x_{i}(t), 1 \leq i \leq$ $N(t)$, with $N(t)$ the number of detections at time $1 \leq t \leq T$ ( $T$ being the length of the time sequence) and assigned each detection to the closest active track at time $t$. Therefore, for each reference track $\theta_{j}^{r}, 1 \leq j \leq\left|\Theta^{r}\right|$, with $\left|\Theta^{r}\right|$ the total number of reference tracks, and for each test track $\theta_{k}^{t}, 1 \leq k \leq\left|\Theta^{t}\right|$, with $\left|\Theta^{t}\right|$ the total number of test tracks, we obtained a set of associated detections. We then considered that a reference track; a test track matched if it shared at least $80 \%$ of common detections with the reference. Finally, for each reference track, we either obtained no test track that matched, exactly one test track that matched or more than one.

\section{b- Synthetic motions}

To validate the robustness and accuracy of $\mathrm{EMC}^{2}$ in different scenarios, we 
simulated three classes of motions: confined diffusion, linear motion and elastic deformation. For confined diffusion, each simulated spot (e.g. fluorescent neuron) can diffuse with coefficient $D=1$ pixe $^{2}$ per frame and is confined to a 10 pixel disk area. For linear motion, each simulated spot moves linearly at speed $v=1$ pixel per frame. When a track reaches the boundary of the field-of-view (a rectangle of 200x200 pixels), it is terminated and another track is initiated at the other side of the FOV. Finally, for elastic deformation, we used the experimental tracks in Hydra to estimate iteratively (i.e. from one frame to the following one) the local deformation for each synthetic track position.

\section{c- Firing rates of individual neurons}

To model the stochastic firing rates of individual neurons (total number of neurons $\left.n_{\text {neurons }}\right)$, we first determined a proportion $\left(\alpha_{\text {stable }}\right)$ of stable spots, i.e. non-blinking cells, with constant fluorescent intensity. In Hydra, stable cells typically correspond to nematocytes or other cell types that also express fluorescent proteins after the genetic editing of the animal, but that don't fire as neurons do [5]. To simulate the correlated activity patterns observed in Hydra, we then divided the $\left(1-\alpha_{\text {stable }}\right) n_{\text {neurons }}$ firing neurons, with intermittent activity and detectability, into $n_{\text {group }}$ ensembles. All neurons in each ensemble fire simultaneously with Poisson rate $\lambda_{\text {group }}=$ size $_{\text {group }} \lambda_{\text {individual }}$, with $\lambda_{\text {individual }}$ the firing rate of individual neurons and size $e_{\text {group }}=(1-$ $\left.\alpha_{\text {stable }}\right) n_{\text {neurons }} / n_{\text {group }}$ the number of neurons in each group. Parameters for each simulation used for the validation of $\mathrm{EMC}^{2}$ algorithm are summarized in Table 3.

\section{d- Generation of synthetic images}

To generate synthetic fluorescence time-lapse sequences, we used a mixed Poisson-Gaussian model [25]. In this model, the intensity $I[x, y]$ at pixel location $[x, y]$ is equal to $I[x, y]=U[x, y]+N\left(0, \sigma_{n}\right)$ where $U$ is a random Poisson variable and $N\left(0, \sigma_{n}\right)$ is additive white Gaussian noise with standard deviation $\sigma_{n}$. The intensity $\lambda[x, y]$ of the Poisson variable varies spatially because it depends on the presence or not of particle spots (neurons). Therefore, $\lambda[x, y]=P[x, y]+B$ with $B$ a constant background value and $P[x, y]$ the spots' intensity at position $[x, y]$. Assuming an additive model for the intensity of the spots, $P[x, y]=\sum_{i=1 . . n_{\text {neurons }}} P_{i}[x, y]$, where $P_{i}[x, y]$ is the signal originating from the $i^{\text {th }}$ spot. We approximated the point-spread- 
function (PSF) of the microscope with a Gaussian profile. Thus, for a particle located at position $\left[x_{0}^{i}, y_{0}^{i}\right]$, its intensity at position $[x, y]$ is given by $P_{i}[x, y]=$ $A_{i} \exp -\frac{\left(x-x_{0}^{i}\right)^{2}+\left(y-y_{0}^{i}\right)^{2}}{2 \sigma_{P S F}^{2}}$, with $A_{i}$ the amplitude of the $i^{\text {th }}$ particle and $\sigma_{P S F}$ the standard deviation of the 2D Gaussian profile of the PSF. We chose a constant amplitude for each particle $A=A_{i}$, for all $1 \leq i \leq n_{\text {neurons. }}$. Parameters used in simulations are summarized in Table 3.

\section{e- Fluorescence kinetics}

When a neuron fires at time $t_{0}$, we model its fluorescence time course with the general kinetics equation

$$
f(t)=A \frac{\exp \left(-\left(\frac{t-t_{0}}{\tau_{\text {decay }}}\right)^{\beta}\right)}{1+\exp \left(-\frac{t-t_{0}-\mu}{\tau_{\text {rise }}}\right)},
$$

where the numerator models a power-law exponential decay of the fluorescence $(\beta=$ 1 models a standard single exponential decay), with a decay time constant $\tau_{\text {decay }}$, and the denominator models a sigmoidal increase of fluorescence with median $\tau_{\text {rise }}$ and time constant $\mu$. Kinetics parameters for each synthetic simulation are summarized in Table 3 . These parameters were obtained by fitting $n=3075$ individual spikes from 444 individual neuron tracks in an experimental time-lapse sequence (250 frames at $10 \mathrm{~Hz}$ ) of GCAMP-labeled Hydra [5] (Supplementary Figure 1). We highlight that, for Hydra elastic simulations, we used a long decay time constant and a power index $\beta=$ 2 instead of 1 for standard confined diffusion and linear motion simulations.

\section{4- Tracking and analyzing single neuron activity in living animals}

\section{a- Two-photon calcium imaging of mouse visual cortex}

Movies used in this study are issued from [33], and experimental protocol for two-photon volumetric imaging of targeted brain regions in mouse visual cortex can be found in the Methods section of this manuscript.

\section{b- Hydra Maintenance}


bioRxiv preprint doi: https://doi org/10.1101/2020.06.22.165696; this version posted March 23, 2021. The copyright holder for this preprint (which was not certified by peer review) is the author/funder, who has granted bioRxiv a license to display the preprint in perpetuity. It is made available under aCC-BY-NC-ND 4.0 International license.

Lagache et al., p.29

Hydra were cultured using standard methods [46] in Hydra medium at $18^{\circ} \mathrm{C}$ in the dark. They were fed freshly hatched Artemia nauplii twice per week.

\section{c- Hydra Imaging}

Transgenic Hydra expressing GCaMP6s in the interstitial cell lineage were used and prepared for imaging studies as previously described [5]. Calcium imaging was performed using a custom spinning disc confocal microscope (Solamere Yokogawa CSU-X1). Samples were illuminated with a $488 \mathrm{~nm}$ laser (Coherent OBIS) and emission light was detected with an ICCD camera (Stanford Photonics XR-MEGA10). Images were captured with a frame rate of 10 frames per second using either a 6X objective (Navitar HRPlanApo 6X/0.3) or a 10X objective (Olympus UMPlanFI 10x/0.30 W).

All movies used in this study can be downloaded from the BioStudies website https://www.ebi.ac.uk/biostudies/studies/S-BSST428.

\section{d- Extracting single neuron activity}

We extracted the fluorescence trace of each individual neuron using the Track Processor Intensity profile within the TrackManager plugin in Icy [20] (see step-by-tep Supplementary tutorial for tracking neurons and exporting track intensity with Icy). For each detection within the track, the extracted intensity corresponded to the mean intensity over a disk centered at the detection's position, with a 2 pixel diameter. When detections are missing (tracking gap), the intensity was set to 0 . Then, for each individual neuron, we denoised its non-zero fluorescence trace using wavelet denoising (wdenoise) in Matlab. We then automatically extracted spikes with a custom procedure where we first computed the discrete derivative of the smoothed fluorescence signal, then set to 0 all negative variations and finally, we detected significant positive variations of the signal (discrete positive derivative $>$ quantile at $98 \%$ of all empirical positive variations) that putatively corresponded to spikes.

\section{e- Statistical characterization of neuronal ensembles in Hydra}

Neuronal ensembles are groups of neurons that repeatedly fire together. Therefore, the activity of neuronal ensembles can be detected as significant co-activity peaks in the raster plot of single neuron firing. To detect significant peaks of activity, we applied 
the procedure described in [37], and identified as peaks times at which the sum of single neuron activity within a time step of $100 \mathrm{~ms}$ fell within the quantile at $0.999 \%$ obtained empirically by circularly shuffling the individual spikes in the activity raster plot.

Then, to relate detected peaks of activity to putative neuronal ensembles, we constructed a vector describing the activity of each individual neuron at the detected peaks with entries 1 if the neuron fires at that peak, and 0 otherwise. We then computed the similarity between these vectors for each of the activity peaks, using the Jaccard index:

$$
J \text { accard }(\text { peak } i \text {, peak } j)=\frac{\text { number of neurons that fire at peak } i \text { and peak } j}{\text { number of neurons that fire at peak } i \text { or peak } j} \text {. }
$$

Then, to estimate the number of neuronal ensembles underlying the detected peaks of activity, we clustered the peaks with a k-means algorithm based on their similarity for different numbers of classes (from 1 to 5 classes typically). K-means clustering was performed using the cosine distance. The optimal number of classes in the k-means clustering algorithms, and therefore the putative number of neuronal ensembles, was computed using the Silhouette index [38]. For a clustering of $N$ peaks into $k$ classes, the Silhouette index is given by $\operatorname{Silhouette}(k)=\frac{1}{N} \sum_{i=1}^{N}\left(b_{i}-a_{i}\right) / \max \left(a_{i}, b_{i}\right)$ where $a_{i}$ is the average distance from the $i^{\text {th }}$ peak to the other peaks in the same cluster as $i$, and $b_{i}$ is the minimum average distance from the $i^{\text {th }}$ peak to peaks in a different cluster, minimized over clusters. An advantage of the Silhouette evaluation criterion over other clustering criteria is its versatility, as it can be used with any distance (cosine distance was used here for the k-means clustering). Finally, we assigned each individual neuron to an ensemble if that neuron fired in more than $50 \%$ of the activity peaks of the identified ensemble. 
Acknowledgements: This work was supported by the NSF (CRCNS 1822550) and the NINDS (R01NS110422; R34NS116740). T.L. was partly supported by the Fondation pour la Recherche Médicale and the Philippe Foundation. A.H. is supported by the NIMH (T32MH018870). MBL research was supported by competitive fellowship funds from the H. Keffer Hartline, Edward F. MacNichol, Jr. Fellowship Fund, The E. E. Just Endowed Research Fellowship Fund, Lucy B. Lemann Fellowship Fund, Frank R. Lillie Fellowship Fund Fellowship Fun, Fries Trust Research Award, Hartline MacNichol Research Award, L. \& A. Colvin Summer Research Fellowship, and John M. Arnold Fellowship Research Award of the Marine Biological Laboratory in Woods Hole, MA. We thank John Wang for help, lab members, MBL staff and members of the MBL Hydra lab for support and advice.

Competing Financial Interest: Authors declare no competing financial interests pertaining to this study.

Author Contributions: T.L., A.F. and R.Y. conceived the project. T.L developed and implemented the method. A.H. generated the genetically modified Hydra line, and acquired confocal time-lapse sequences. T.L analyzed data and wrote the manuscript. All authors edited the manuscript. R.Y assembled and directed the team, providing guidance and funding. 
Table 1: A review of SPT methods in bio-imaging

\begin{tabular}{|c|c|c|c|c|c|c|c|c|}
\hline Algorithm & Type & Detection & Linking & $\begin{array}{c}\text { Gap } \\
\text { closing }\end{array}$ & Pros & Cons & Freely available & Ref. \\
\hline Mosaic & $\begin{array}{l}\text { Detect \& } \\
\text { Link }\end{array}$ & $\begin{array}{l}\text { Gaussian Convolution \& } \\
\text { Thresholding }\end{array}$ & $\begin{array}{l}\text { Global distance } \\
\text { minimization }\end{array}$ & Yes & $\begin{array}{l}\text { Fast. Accounts for spot } \\
\text { intensity and size in } \\
\text { distance computation. }\end{array}$ & $\begin{array}{l}\text { Gap closing does not handle large } \\
\text { motion. Not robust in very } \\
\text { cluttered conditions. }\end{array}$ & $\begin{array}{l}\text { Particle Tracker } \\
\text { plugin (ImageJ) }\end{array}$ & {$[26]$} \\
\hline Sage et al. & Global & None & Energy minimization & Yes & Global. & $\begin{array}{l}\text { Designed for one or few sparse } \\
\text { particles }\end{array}$ & ImageJ plugin & [47] \\
\hline Bonneau et al. & Global & None & Energy minimization & Yes & $\begin{array}{l}\text { Global. Robust gap } \\
\text { closing with minimal- } \\
\text { path algorithm }\end{array}$ & $\begin{array}{l}\text { Designed for few sparse particles. } \\
\text { High computational load. }\end{array}$ & No & [48] \\
\hline TrackMate & $\begin{array}{l}\text { Detect \& } \\
\text { Link }\end{array}$ & $\begin{array}{l}\text { Wavelet transformation or } \\
\text { Gaussian convolution \& } \\
\text { thresholding }\end{array}$ & $\begin{array}{c}\text { Global distance } \\
\text { minimization }\end{array}$ & Yes & $\begin{array}{l}\text { Fast. Handles split \& } \\
\text { merge events. }\end{array}$ & $\begin{array}{l}\text { Gap closing does not handle } \\
\text { large, non-linear motion. Many } \\
\text { user-defined parameters }\end{array}$ & $\begin{array}{l}\text { TrackMate plugin } \\
\quad \text { (ImageJ) }\end{array}$ & {$[27]$} \\
\hline eMHT & $\begin{array}{l}\text { Detect \& } \\
\text { Link }\end{array}$ & $\begin{array}{c}\text { Wavelet transformation \& } \\
\text { thresholding }\end{array}$ & $\begin{array}{c}\text { Probabilistic (Multiple } \\
\text { Hypothesis) }\end{array}$ & Yes & $\begin{array}{l}\text { Robust in cluttered } \\
\text { environment. Few user- } \\
\text { defined parameters }\end{array}$ & $\begin{array}{l}\text { Slower than global distance } \\
\text { minimization. Cannot close large } \\
\text { gaps }(>\sim 5 \text { frames) due to } \\
\text { computational load }\end{array}$ & $\begin{array}{l}\text { Spot Tracking plugin } \\
\text { (Icy) }\end{array}$ & {$[24]$} \\
\hline NeRVE & $\begin{array}{l}\text { Detect \& } \\
\text { Link }\end{array}$ & Watershed Segmentation & Point-set registration & No & $\begin{array}{c}\text { Robust to dense } \\
\text { packing of particles. } \\
\text { Handles non-linear } \\
\text { deformations. }\end{array}$ & $\begin{array}{c}\text { High computational load. Not } \\
\text { robust to many missing detections } \\
\text { and long gaps in highly deforming } \\
\text { environments. }\end{array}$ & Matlab GUI & [10] \\
\hline MAP-4D-DAE & $\begin{array}{l}\text { Detect \& } \\
\text { Link }\end{array}$ & Not specified & $\begin{array}{l}\text { Probabilistic (Multiple } \\
\text { Hypothesis) }+ \\
\text { Autoencoding for } \\
\text { particle motion } \\
\text { modeling }\end{array}$ & Yes & $\begin{array}{l}\text { Robust in cluttered } \\
\text { environment. Few user- } \\
\text { defined parameters. } \\
\text { Handles non-linear } \\
\text { deformations }\end{array}$ & $\begin{array}{l}\text { Slower than global distance } \\
\text { minimization. Cannot close large } \\
\text { gaps (> } \sim 5 \text { frames) due to } \\
\text { computational load }\end{array}$ & No & $\begin{array}{r}{[39} \\
40]\end{array}$ \\
\hline
\end{tabular}


Table 2: List of tested tracking algorithms in manual validation

\begin{tabular}{|c|c|c|c|c|}
\hline Name & $\begin{array}{c}\text { Local association of detected } \\
\text { particles }\end{array}$ & $\begin{array}{c}\text { Elastic Motion } \\
\text { Correction ? }\end{array}$ & Gap closing & $\%$ match \\
\hline Manual & Bayesian (eMHT) & No & Manual & $100 \%$ (ground truth) \\
\hline EMC $^{2}$ & Bayesian (eMHT) & Yes & GDM & $90.5 \%$ \\
\hline TrackMate & $\begin{array}{c}\text { Global distance minimization } \\
\text { (GDM) }\end{array}$ & No & GDM & $54.6 \%$ \\
\hline $\begin{array}{c}\text { EMC } \\
\text { Correction }\end{array}$ & Bayesian (eMHT) & No & GDM & $54.3 \%$ \\
\hline
\end{tabular}


bioRxiv preprint doi: https://doi.org/10.1101/2020.06.22.165696; this version posted March 23,2021 . The copyright holder for this preprint (which was not certified by peer review) is the author/funder, who has granted bioRxiv a license to display the preprint in perpetuity. It is made available under aCC-BY-NC-ND 4.0 International license.

Lagache et al., p.34

Table 3: Detailed results of statistical extraction of neuronal ensembles in Hydra ( $n=8$ animals)

\begin{tabular}{|c|c|c|c|c|c|c|c|}
\hline Animal & $\begin{array}{l}\text { Movie length } \\
\text { (frames) }\end{array}$ & $\begin{array}{l}\text { Tracks (>150 } \\
\text { frames) }\end{array}$ & $\begin{array}{l}\text { Activity } \\
\text { peaks }\end{array}$ & Ensembles & $C B$ neurons & RP1 neurons & RP2 neurons \\
\hline 1 & 900 & 411 & 11 & 2 & & $139(33.8 \%)$ & $37(9.0 \%)$ \\
\hline 1 & 1636 & 723 & 37 & 3 & $202(27.9 \%)$ & 121(16.7\%) & $49(6.8 \%)$ \\
\hline 1 & 1371 & 607 & 27 & 3 & $222(36.6 \%)$ & $78(12.9 \%)$ & $41(6.8 \%)$ \\
\hline 1 & 988 & 516 & 23 & 3 & $220(42.6 \%)$ & $49(9.5 \%)$ & $55(10.7 \%)$ \\
\hline 2 & 1000 & 531 & 17 & 2 & $34(6.4 \%)$ & $29(5.5 \%)$ & \\
\hline 2 & 1000 & 518 & 17 & 2 & $59(11.4 \%)$ & $29(5.6 \%)$ & \\
\hline 3 & 1000 & 191 & 20 & 2 & $16(8.4 \%)$ & $11(5.8 \%)$ & \\
\hline 4 & 1000 & 243 & 13 & 2 & $16(6.6 \%)$ & $17(7.0 \%)$ & \\
\hline 4 & 1000 & 173 & 16 & 2 & & $24(13.9 \%)$ & $8(4.6 \%)$ \\
\hline 5 & 1000 & 147 & 15 & 2 & $14(9.5 \%)$ & $7(4.8 \%)$ & \\
\hline 6 & 1000 & 379 & 11 & 2 & $14(3.7 \%)$ & $10(2.6 \%)$ & \\
\hline 7 & 1000 & 173 & 9 & 2 & $59(34.1 \%)$ & $25(14.5 \%)$ & \\
\hline 8 & 1000 & 244 & 10 & 2 & $44(18.0 \%)$ & $18(7.4 \%)$ & \\
\hline Mean & 1067 & 374 & 17.3 & & $82(18.7 \%)$ & $43(10.8 \%)$ & $38(7.6 \%)$ \\
\hline $\begin{array}{l}\text { Standard } \\
\text { error }\end{array}$ & 56 & 53 & 2.2 & & $26(4.4 \%)$ & $12(2.3 \%)$ & $8(1.8 \%)$ \\
\hline
\end{tabular}


Table 4: Parameters used for synthetic simulations

\begin{tabular}{|c|c|c|c|c|c|c|c|c|c|c|c|c|c|}
\hline Parameters & $n_{\text {neurons }}$ & $\alpha_{\text {stable }}$ & $n_{\text {group }}$ & $\lambda_{\text {individual }}$ & $A$ & $\tau_{\text {decay }}$ & $\beta$ & $\mu$ & $\tau_{\text {rise }}$ & $\sigma_{P S F}$ & $\sigma_{n}$ & $B$ & $S N R=\frac{A}{B+\sigma_{n}^{2}}$ \\
\hline Name & $\begin{array}{c}\text { Total } \\
\text { number of } \\
\text { neurons }\end{array}$ & $\begin{array}{l}\text { \% of } \\
\text { non- } \\
\text { firing } \\
\text { spots }\end{array}$ & $\begin{array}{c}\text { Number of } \\
\text { neuron } \\
\text { groups }\end{array}$ & $\begin{array}{l}\text { Individual } \\
\text { firing rate }\end{array}$ & Amplitude & $\begin{array}{c}\text { Fluorescence } \\
\text { decay time } \\
\text { constant }\end{array}$ & $\begin{array}{l}\text { Decay } \\
\text { power }\end{array}$ & $\begin{array}{c}\text { Median } \\
\text { rising time }\end{array}$ & $\begin{array}{c}\text { Rising time } \\
\text { constant }\end{array}$ & $\begin{array}{c}\text { St. Dev. of the } \\
\text { PSF }\end{array}$ & $\begin{array}{l}\text { St. Dev. of the } \\
\text { Gaussian } \\
\text { noise }\end{array}$ & $\begin{array}{c}\text { Poisson } \\
\text { background }\end{array}$ & $\begin{array}{l}\text { Signal-to-noise } \\
\text { ratio }\end{array}$ \\
\hline $\begin{array}{c}\text { Confined } \\
\text { diffusion \& } \\
\text { Linear motion }\end{array}$ & 150 & $20 \%$ & 10 & 0.01 frame $^{-1}$ & 100 & 3 frames & 1 & 1 frame & 0.5 frames & 1 pixel & 5 & 10 & $\approx 3$ \\
\hline $\begin{array}{c}\text { Elastic } \\
\text { deformation } \\
\text { (Hydra) }\end{array}$ & 500 & $20 \%$ & 10 & $\begin{array}{l}0.0002 \\
\text { frame }^{-1}\end{array}$ & 100 & 15 frames & 2 & 2 frames & 0.5 frames & 1 pixel & 5 & 10 & $\approx 3$ \\
\hline
\end{tabular}




\section{References}

1. Yuste, R. and L.C. Katz, Control of postsynaptic Ca2+ influx in developing neocortex by excitatory and inhibitory neurotransmitters. Neuron, 1991. 6(3): p. 333-344.

2. Aharoni, D., et al., All the light that we can see: a new era in miniaturized microscopy. Nature methods, 2019. 16(1): p. 11.

3. Yang, W. and R. Yuste, In vivo imaging of neural activity. Nat Methods, 2017. 14(4): p. 349-359.

4. Nguyen, J.P., et al., Whole-brain calcium imaging with cellular resolution in freely behaving Caenorhabditis elegans. Proc Natl Acad Sci U S A, 2016. 113(8): p. E1074-81.

5. Dupre, C. and R. Yuste, Non-overlapping Neural Networks in Hydra vulgaris. Curr Biol, 2017. 27(8): p. 1085-1097.

6. Ahrens, M.B., et al., Whole-brain functional imaging at cellular resolution using lightsheet microscopy. Nature methods, 2013. 10(5): p. 413-420.

7. Fosque, B.F., et al., Labeling of active neural circuits in vivo with designed calcium integrators. Science, 2015. 347(6223): p. 755-760.

8. Han, S., et al., Comprehensive machine learning analysis of Hydra behavior reveals a stable basal behavioral repertoire. Elife, 2018. 7.

9. Bouchard, M.B., et al., Swept confocally-aligned planar excitation (SCAPE) microscopy for high-speed volumetric imaging of behaving organisms. Nature photonics, 2015. 9(2): p. 113-119.

10. Nguyen, J.P., et al., Automatically tracking neurons in a moving and deforming brain. PLoS Comput Biol, 2017. 13(5): p. e1005517.

11. Seelig, J.D., et al., Two-photon calcium imaging from head-fixed Drosophila during optomotor walking behavior. Nature methods, 2010. 7(7): p. 535-540.

12. Kim, D.H., et al., Pan-neuronal calcium imaging with cellular resolution in freely swimming zebrafish. Nature methods, 2017. 14(11): p. 1107-1114.

13. Thevenaz, P., U.E. Ruttimann, and M. Unser, A pyramid approach to subpixel registration based on intensity. IEEE transactions on image processing, 1998. 7(1): p. 27-41.

14. Dubbs, A., J. Guevara, and R. Yuste, moco: Fast motion correction for calcium imaging. Frontiers in neuroinformatics, 2016. 10: p. 6.

15. Lagache, T., et al., Tracking Activity In A Deformable Nervous System With Motion Correction And Point-Set Registration. bioRxiv, 2018: p. 373035.

16. $\mathrm{Yu}, \mathrm{X}$., et al., Fast deep learning correspondence for neuron tracking and identification in C. elegans using synthetic training. arXiv preprint arXiv:2101.08211, 2021.

17. Pnevmatikakis, E.A., et al., Simultaneous Denoising, Deconvolution, and Demixing of Calcium Imaging Data. Neuron, 2016. 89(2): p. 285-99.

18. Nejatbakhsh, A., et al. Demixing Calcium Imaging Data in C. elegans via Deformable Non-negative Matrix Factorization. in International Conference on Medical Image Computing and Computer-Assisted Intervention. 2020. Springer.

19. Vaadia, R.D., et al., Characterization of proprioceptive system dynamics in behaving Drosophila larvae using high-speed volumetric microscopy. Current Biology, 2019.

20. de Chaumont, F., et al., Icy: an open bioimage informatics platform for extended reproducible research. Nat Methods, 2012. 9(7): p. 690-6.

21. Yuste, R., From the neuron doctrine to neural networks. Nature reviews neuroscience, 2015. 16(8): p. 487. 
bioRxiv preprint doi: https://doi org/10.1101/2020.06.22.165696; this version posted March 23, 2021. The copyright holder for this preprint (which was not certified by peer review) is the author/funder, who has granted bioRxiv a license to display the preprint in perpetuity. It is made available under aCC-BY-NC-ND 4.0 International license.

22. Smal, I., et al., Quantitative comparison of spot detection methods in fluorescence microscopy. IEEE Trans Med Imaging, 2010. 29(2): p. 282-301.

23. Ruusuvuori, P., et al., Evaluation of methods for detection of fluorescence labeled subcellular objects in microscope images. BMC Bioinformatics, 2010. 11: p. 248.

24. Chenouard, N., I. Bloch, and J.C. Olivo-Marin, Multiple hypothesis tracking for cluttered biological image sequences. IEEE Trans Pattern Anal Mach Intell, 2013. 35(11): p. 27363750.

25. Chenouard, N., et al., Objective comparison of particle tracking methods. Nat Methods, 2014. 11(3): p. 281-9.

26. Sbalzarini, I.F. and P. Koumoutsakos, Feature point tracking and trajectory analysis for video imaging in cell biology. J Struct Biol, 2005. 151(2): p. 182-95.

27. Jaqaman, K., et al., Robust single-particle tracking in live-cell time-lapse sequences. Nat Methods, 2008. 5(8): p. 695-702.

28. Tinevez, J.Y., et al., TrackMate: An open and extensible platform for single-particle tracking. Methods, 2017. 115: p. 80-90.

29. Arhel, N., et al., Quantitative four-dimensional tracking of cytoplasmic and nuclear HIV1 complexes. Nature methods, 2006. 3(10): p. 817.

30. Genovesio, A., et al., Multiple particle tracking in 3-D+t microscopy: method and application to the tracking of endocytosed quantum dots. IEEE Transactions on Image Processing, 2006. 15(5): p. 1062-1070.

31. Arhel, N., et al., Quantitative four-dimensional tracking of cytoplasmic and nuclear HIV1 complexes. Nat Methods, 2006. 3(10): p. 817-24.

32. Chui, H.L. and A. Rangarajan, $A$ new algorithm for non-rigid point matching. leee Conference on Computer Vision and Pattern Recognition, Proceedings, Vol li, 2000: p. 44-51.

33. Pérez-Ortega, J., T. Alejandre-García, and R. Yuste, Long-term stability of neuronal ensembles in mouse visual cortex. bioRxiv, 2020.

34. Ober, R.J., S. Ram, and E.S. Ward, Localization accuracy in single-molecule microscopy. Biophysical journal, 2004. 86(2): p. 1185-1200.

35. Buzsáki, G., Large-scale recording of neuronal ensembles. Nature neuroscience, 2004. 7(5): p. 446-451.

36. Passano, L. and C. McCullough, The light response and the rhythmic potentials of Hydra. Proceedings of the National Academy of Sciences of the United States of America, 1962. 48(8): p. 1376.

37. Carrillo-Reid, L., et al., Endogenous sequential cortical activity evoked by visual stimuli. Journal of Neuroscience, 2015. 35(23): p. 8813-8828.

38. Rousseeuw, P.J., Silhouettes: a graphical aid to the interpretation and validation of cluster analysis. Journal of computational and applied mathematics, 1987. 20: p. 5365.

39. Yao, Y., et al., Deep-learning method for data association in particle tracking. Bioinformatics, 2020. 36(19): p. 4935-4941.

40. Spilger, R., et al., A Recurrent Neural Network for Particle Tracking in Microscopy Images Using Future Information, Track Hypotheses, and Multiple Detections. IEEE Transactions on Image Processing, 2020. 29: p. 3681-3694.

41. Beatson, R., M.J.D. Powell, and A. Tan, Fast evaluation of polyharmonic splines in three dimensions. IMA Journal of Numerical Analysis, 2007. 27(3): p. 427-450. 
42. Kuhn, H.W., The Hungarian method for the assignment problem. Naval research logistics quarterly, 1955. 2(1 - 2): p. 83-97.

43. Jonker, R. and T. Volgenant, Improving the Hungarian assignment algorithm. Operations Research Letters, 1986. 5(4): p. 171-175.

44. Olivo-Marin, J.C., Extraction of spots in biological images using multiscale products. Pattern Recognition, 2002. 35(9): p. 1989-1996.

45. Zhang, B., J. Zerubia, and J.-C. Olivo-Marin, Gaussian approximations of fluorescence microscope point-spread function models. Applied optics, 2007. 46(10): p. 1819-1829.

46. Lenhoff, H.M. and R.D. Brown, Mass culture of hydra: an improved method and its application to other aquatic invertebrates. Laboratory Animals, 1970. 4(1): p. 139-154.

47. Sage, D., et al., Automatic tracking of individual fluorescence particles: application to the study of chromosome dynamics. IEEE Trans Image Process, 2005. 14(9): p. 137283.

48. Bonneau, S., M. Dahan, and L.D. Cohen, Single quantum dot tracking based on perceptual grouping using minimal paths in a spatiotemporal volume. IEEE Trans Image Process, 2005. 14(9): p. 1384-95. 